\title{
A Theoretical Analysis of Rheodielectric Response of Type-A Polymer Chains
}

\author{
TAKASHI UNEYAMA, ${ }^{1}$ YUICHI MASUBUCHI, ${ }^{1}$ KAZUSHI HORIO, ${ }^{1}$ YUMI MATSUMIYA, ${ }^{1}$ HIROSHI WATANABE, ${ }^{1}$ \\ JAI A. PATHAK, ${ }^{2}$ C. MICHAEL ROLAND ${ }^{2}$ \\ ${ }^{1}$ Institute for Chemical Research, Kyoto University, Uji, Kyoto 611-0011, Japan \\ ${ }^{2}$ U.S. Naval Research Laboratory, Chemistry Division, Code 6120, Washington DC 20375-5342
}

Received 7 January 2009; revised 27 February 2009; accepted 9 March 2009

DOI: 10.1002 / polb.21708

Published online in Wiley InterScience (www.interscience.wiley.com).

\begin{abstract}
For type-A polymer chains having type-A dipoles parallel along the chain backbone (such as cis-polyisoprene), a theoretical analysis was conducted for the rheodielectric response to relate this response to the chain dynamics. The rheodielectric response in the shear gradient direction ( $y$ direction) under steady shear was analyzed on the basis of a Langevin equation. It turned out that the relaxation time is exactly the same for the rheodielectric relaxation function and the end-to-end vector autocorrelation function defined in the shear gradient direction and that the relaxation mode distribution also coincides for these functions at least up to second order of the shear rate (corresponding to the lowest order of nonlinearities of these functions). Consequently, the Green-Kubo theorem holds satisfactorily, and the rheodielectric intensity is proportional to the squared chain size in $y$ direction, $\left\langle R_{y}^{2}\right\rangle$, averaged over the time-independent conformational distribution function under steady shear. The situation is more complicated under large amplitude oscillatory strain (LAOS) because the conformational distribution function $f_{\text {LAOS }}$ is synchronized with LAOS to oscillate at the LAOS frequency, $\Omega$. The rheodielectric response under LAOS was found to detect this oscillation of $f_{\text {LAOS }}$ being coupled with the oscillation of the electric field, $E(t)=E_{0} \sin \omega t$, and thus, split into a series of components oscillating at frequencies $\omega$ and $\omega \pm \beta \Omega(\beta=1,2, \ldots)$. Consequently, the rheodielectric intensity under LAOS, evaluated from the component oscillating at $\omega$, is no longer proportional to $\left\langle R_{y}^{2}\right\rangle$. However, the relative mode distribution and relaxation time of this component can be directly related to those of the end-to-end vector correlation averaged over a nonoscillatory part of $f_{\text {LAOS. }}$ (C) 2009 Wiley Periodicals, Inc. J Polym Sci Part B: Polym Phys 47: 1039-1057, 2009

Keywords: cis-polyisoprene; cooperative effects; coupling; dielectric properties; large amplitude oscillatory shear (LAOS); rheodielectric behavior; steady shear flow; structure-property relations; type-A chain
\end{abstract}

\section{INTRODUCTION}

Dynamics in soft materials has been experimentally investigated with a variety of spectroscopies

Correspondence to: H. Watanabe (E-mail: hiroshi@scl. kyoto-u.ac.jp)

Journal of Polymer Science: Part B: Polymer Physics, Vol. 47, 1039-1057 (2009) (C) 2009 Wiley Periodicals, Inc. such as the viscoelastic, NMR, and dielectric methods. The same microscopic dynamics is differently reflected/averaged in different properties. ${ }^{1,2}$ For example, for so-called type-A polymers having the electric dipoles parallel along the chain backbone, the linear viscoelastic and dielectric properties in long time scales reflect the isochronal orientational anisotropy and orientational memory of the chain, respectively. ${ }^{1,2}$ These 
properties are commonly governed by the globalchain dynamics, but their details (such as the relaxation mode distribution) are not identical to each other because of the difference between the isochronal anisotropy and the memory, the latter detecting the orientational correlation at two separate times (e.g., 0 and $t)^{1}{ }^{1}$

Thus, details of the dynamics are better understood if we measure different properties of a given material simultaneously. The well-known example of this simultaneous measurement is the rheooptical measurement, ${ }^{1,2}$ which often enables the molecular assignment of a specific relaxation process. The other example is the rheodielectric measurement in which the dielectric response of a material under flow/deformation is detected together with the mechanical response.

Rheodielectric studies have been conducted for a variety of materials ${ }^{3-12}$ that include low molecular weight liquid crystalline (LC) ${ }^{4(a, b), 5,12}$ carbon black (CB) suspensions, ${ }^{6}$ polyelectrolytes (polymer/salt composites), ${ }^{9}$ and entangled polymer melts/solutions. ${ }^{3,5,7,8,10-12}$ In heterogeneous materials, higher order structures tend to be distorted/ disrupted even by slow flow that does not affect the dielectrically active molecular motion itself. For this case, the rheodielectric change just detects the structural changes (affecting the direction of molecular motion/current conduction) such as the orientation of LC textures ${ }^{4(a, b)}$ and scission of $\mathrm{CB}$ network. ${ }^{6}$ In contrast, the rheodielectric effect for homogeneous systems usually detects changes of the molecular motion due to flow/deformation. This effect needs to be analyzed on the basis of a theoretical expression of the dielectric relaxation function $\Phi(t)$ that relates the rheodielectric response to the molecular dynamics. In this paper, we focus on this expression for homogeneous systems of a representative type-A polymer having rather small type-A dipoles, cis-1,4polyisoprene (PI).

For PI at equilibrium, the Green-Kubo theorem $^{13-15}$ unequivocally leads to the Cole expres$\operatorname{sion}^{13}$ in which $\Phi(t)$ is given as the autocorrelation of the end-to-end dipole averaged over the equilibrium distribution function of the chain conformation. Previous work ${ }^{3,7}$ considered that $\Phi(t)$ of PI under steady shear is similarly expressed as the autocorrelation averaged over the time-independent distribution function under shear given that local equilibrium is achieved, but this expression was not rigorously proved. Under large amplitude oscillatory shear (LAOS), the same expression has been utilized ${ }^{11,12}$ without any justification.
This paper attempts to theoretically analyze the rheodielectric response of PI under steady shear and LAOS and relate the response to the conformational dynamics of the PI chains. For this purpose, we consider only the fluctuation of permanent type-A dipoles. (The instantaneous responses reflecting the atomic/electronic polarization as well as the local segmental motion can be simply added to the resulting dielectric function.) For convenience of this analysis, we first revisit the known derivation of the Cole expression for $\Phi(t)$ at equilibrium. ${ }^{13}$ Then, we examine if the same expression is valid under steady shear flow and LAOS. Readers familiar with the Cole expression can skip the first part of the next section.

\section{RESULTS AND DISCUSSION}

\section{Dielectric Signal at Equilibrium (Cole Expression) ${ }^{13}$}

We consider an equilibrium system of type-A chains (e.g., PI) that have just a small type-A dipole moment, $\tilde{\mu}$ per unit length of the chain backbone, and exhibit negligibly small dipoledipole interaction $\left(\tilde{\mu}^{2} \ll k_{\mathrm{B}} T\right.$ with $k_{\mathrm{B}}=$ Boltzmann constant and $T=$ absolute temperature). We apply a weak, time $(t)$ dependent electric field $E(t)$ in $y$ direction and detect the macroscopic polarization $P(t)$ in the same direction. The end-to-end dipole of the $j$ th chain in this direction is given by $\tilde{\mu} R_{y, j}$ with $R_{y, j}$ being the $y$ component of the end-to-end vector of this chain. $P(t)$ of our interest is given by a sum of these dipoles being averaged over a conformational distribution function $f(\mathbf{p}, \mathbf{q}, t)$ in the presence of the electric field, where $f$ is defined in the phase space specified by generalized momentum $\mathbf{p}$ and coordinate q. As explained in Appendix A, we can express the time evolution of $f(\mathbf{p}, \mathbf{q}, t)$ in terms of the time-independent equilibrium distribution function $f^{\circ}(\mathbf{p}, \mathbf{q})$ (in the absence of the electric field) and the Hamiltonian of the system to find an explicit expression of $P(t)$,

$$
\begin{aligned}
P(t)= & \frac{\tilde{\mu}^{2}}{k_{\mathrm{B}} T} \int_{-\infty}^{t} \mathrm{~d} s E(s) \int \mathrm{d} \mathbf{p d} \mathbf{q}\left\{\Sigma_{j} R_{y, j}(t)\right\} \\
& \times \frac{\mathrm{d}}{\mathrm{d} s}\left\{\Sigma_{j} R_{y_{j}}(s)\right\} f^{\mathrm{o}}(\mathbf{p}, \mathbf{q}) \\
& =\frac{v \tilde{\mu}^{2}}{k_{\mathrm{B}} T} \int_{-\infty}^{t} \mathrm{~d} s E(s)\left\langle R_{y}(t) \frac{\mathrm{d} R_{y}(s)}{\mathrm{d} s}\right\rangle_{\mathrm{eq}}
\end{aligned}
$$

where $v$ denotes the chain number density and $\langle\ldots\rangle_{\mathrm{eq}} \equiv \int \mathrm{d} \mathbf{p} \mathrm{d} \mathbf{q} \ldots f^{\circ}(\mathbf{p}, \mathbf{q})$. Since the system is 
stationary at equilibrium, that is, $\langle A(t) B(s)\rangle_{\mathrm{eq}}=$ $\langle A(t-s) B(0)\rangle_{\mathrm{eq}}$ and $\langle A(t)\{\mathrm{d} B(s) / \mathrm{d} s\}\rangle_{\mathrm{eq}}=-\partial\langle A(t-s)$ $B(0)\rangle_{\mathrm{eq}} / \partial t$ for any microscopic quantities $A$ and $B$, eq 1 reduces to

$$
\begin{gathered}
P(t)=-\frac{v \tilde{\mu}^{2}}{k_{\mathrm{B}} T}\left\langle R_{y}^{2}\right\rangle_{\mathrm{eq}} \int_{-\infty}^{t} \mathrm{~d} s E(s) \frac{\partial \Phi(t-s)}{\partial t} \\
\text { with } \Phi(t)=\frac{\left\langle R_{\mathrm{y}}(t) R_{\mathrm{y}}(0)\right\rangle_{\mathrm{eq}}}{\left\langle R_{\mathrm{y}}^{2}\right\rangle_{\mathrm{eq}}}
\end{gathered}
$$

$\Phi(t)$ is the normalized dielectric relaxation function $(\Phi(0)=1)$ that detects the autocorrelation of the end-to-end vector of the PI chains at equilibrium.

The dynamic dielectric constant (or dielectric permittivity) $\varepsilon^{\prime}(\omega)$ and dielectric loss $\varepsilon^{\prime \prime}(\omega)$ under the small oscillatory electric field, $E(t)=E_{0} \sin \omega t$, are related to $\Phi(t)$ as

$$
\begin{array}{r}
\varepsilon^{\prime}(\omega)=\varepsilon^{\prime}(\infty)-\Delta \varepsilon \int_{0}^{\infty} \mathrm{d} t^{\prime} \cos \omega t^{\prime} \frac{\mathrm{d} \Phi\left(t^{\prime}\right)}{\mathrm{d} t^{\prime}} \\
\varepsilon^{\prime \prime}(\omega)=-\Delta \varepsilon \int_{0}^{\infty} \mathrm{d} t^{\prime} \sin \omega t^{\prime} \frac{\mathrm{d} \Phi\left(t^{\prime}\right)}{\mathrm{d} t^{\prime}}
\end{array}
$$

with

$$
\Delta \varepsilon=F\left(\frac{4 \pi \tilde{\mu}^{2}}{k_{\mathrm{B}} T}\right) v\left\langle R_{y}^{2}\right\rangle_{\mathrm{eq}} \quad(\text { for PI })
$$

In eq $3 \mathrm{a}$, we have introduced the high-frequency dielectric constant reflecting the atomic/electronic polarization and the local segmental motion, $\varepsilon^{\prime}(\infty)$, not considered in derivation of eq 1 . In addition, in eq 4, we have introduced a correction factor for the internal electric field $F$ ( $\cong 1$ for the global relaxation of $\mathrm{PI}^{16}$ ) as well as the prefactor of $4 \pi$ (in MKSA units).

\section{Dielectric Signal from PI under Steady Shear}

The macroscopic polarization $P(t)$ of type-A chains under steady shear can be expressed in terms of the end-to-end dipoles $\tilde{\mu} R_{y, j}$ and the conformational distribution function $f(\mathbf{p}, \mathbf{q}, t)$ in the presence of the electric field, as similar to the situation at equilibrium. However, the time evolution of $f(\mathbf{p}, \mathbf{q}, t)$ under steady shear is affected by advection $^{15,17}$ and we cannot rigorously derive the expression of $P(t)$ in a way explained in Appendix
A. In fact, the nonequilibrium statistical analysis by Evans et al. ${ }^{17-19}$ suggests that the Green-Kubo theorem, showing the coincidence of the macroscopic response function and the autocorrelation function (cf. eq 1), is valid under steady shear for thermally driven heat conduction and particle diffusion but not for transport phenomena driven by a vectorial field. ${ }^{17-19}$ Since the rheodieletric response belongs to the latter case, the GreenKubo theorem would not be valid rigorously for this response. Furthermore, the entropy production under steady shear ${ }^{20,21}$ would also result in this lack of rigorous validity.

In this situation, it is very important to quantify differences between the relaxation time/mode distribution of the rheodielectric relaxation function (measured for $\mathrm{PI}^{7,8,10}$ ) and those of the endto-end vector autocorrelation function. For this purpose, this section utilizes a Langevin equation to make a kinematic analysis of the rheodielectric response. This analysis is equivalent to an analysis based on the Hamiltonian and distribution function (made in Appendix A), but the results are easier to discuss for the Langevin analysis.

\section{Langevin Analysis}

We consider an ensemble of type-A linear chains, for example, PI, each being composed of $N+1$ segments and having the dipole moment $\tilde{\mu}$ per unit length of the backbone. (We consider a case of $N \gg 1$ and do not distinguish $N$ and $N+1$.) Since this chain is equivalent to a totally neutral chain having negative and positive charges $-\tilde{\mu}$ and $\tilde{\mu}$ at zero-th and $N$ th segments, the force acting on $n$th segment of the chain due to a constant electric field $E$ in the $y$ direction can be expressed as

$$
\mathbf{F}^{\mathrm{E}}(n, t)=\left[\begin{array}{c}
0 \\
\tilde{\mu} E\{\delta(n-N)-\delta(n)\} \\
0
\end{array}\right]
$$

The time evolution of the position $\mathbf{r}(n, t)$ of $n$th segment is determined by the Langevin equation describing a balance of the frictional force, intrachain and interchain potential forces, the Brownian force, and this $\mathbf{F}^{\mathrm{E}}(n, t)$. Within the mean-field treatment where the interactions for a given chain from the surrounding chains are preaveraged, this equation can be cast in a general form,

$$
\begin{array}{r}
-\zeta\{\dot{\mathbf{r}}(n, t)-\dot{\boldsymbol{\Gamma}} \cdot \mathbf{r}(n, t)\}+\boldsymbol{\Lambda}(n ; \dot{\gamma}) \cdot \mathbf{r}(n, t) \\
+\mathbf{F}^{\mathrm{B}}(n, t)+\mathbf{F}^{\mathrm{E}}(n, t)=\mathbf{0}
\end{array}
$$

with 


$$
\dot{\boldsymbol{\Gamma}}=\left[\begin{array}{lll}
0 & \dot{\gamma} & 0 \\
0 & 0 & 0 \\
0 & 0 & 0
\end{array}\right] \quad \text { (shear rate tensor) }
$$

In eq $6, \zeta$ is the segmental friction coefficient and $\mathbf{F}^{\mathrm{B}}(n, t)$ is the Brownian force that can be safely assumed to have a white-noise character, $\left\langle\mathbf{F}^{\mathrm{B}}(n\right.$, $\left.t) \mathbf{F}^{\mathrm{B}}\left(n^{\prime}, t^{\prime}\right)\right\rangle=2 \mathbf{I} \zeta k_{\mathrm{B}} T \delta\left(n-n^{\prime}\right) \delta\left(t-t^{\prime}\right)$ with $\mathbf{I}=$ unit tensor. In general, $\boldsymbol{\Lambda}(n ; \dot{\gamma})$ is a three-dimensional tensorial operator with respect to $n$ (having components $\boldsymbol{\Lambda}_{\alpha \beta}$ with $\alpha, \beta=x, y$, and $z$ ) and may explicitly depend on the shear rate $\dot{\gamma}$. This $\Lambda$, giving the potential forces for a given chain conformation $\mathbf{r}(n, t)$ thereby governing the chain motion, is determined by an effective Hamiltonian of the chain under steady shear.

The actual chain motion can be decomposed into eigenmodes, as noted from experiments for a special type of linear PI having inverted type-A dipoles. ${ }^{1,22-24}$ Thus, we may safely assume that $\boldsymbol{\Lambda}$ is a linear operator associated with the eigenmodes. In fact, the dielectric mode distribution of linear PI hardly changes under the steady shear conditions examined so far, ${ }^{7}$ suggesting that the eigenmodes are well defined and $\boldsymbol{\Lambda}$ is linear under these conditions. (For completeness, the other type of Langevin analysis without the assumption of the linearity of $\boldsymbol{\Lambda}$ is later explained in Appendix $\mathrm{C}$, although this analysis cannot give explicitly the dielectric relaxation time/intensity.)

On application of the constant electric field $E$ at $t=0$ in the shear gradient $(y)$ direction, the polarization of the steadily sheared type-A chains grows in this direction with time. The unnormalized dielectric relaxation function characterizing this growth is given by $\Phi_{\mathrm{ss}}^{\mathrm{un}}(t ; \dot{\gamma})=(\tilde{\mu} v / E)\left(\left\langle R_{y}(\infty)\right\rangle_{\mathrm{ss}}^{E}\right.$ $\left.-\left\langle R_{y}(t)\right\rangle_{\mathrm{ss}}^{E}\right)$, where $\left\langle R_{y}(t)\right\rangle_{\mathrm{ss}}^{E}$ is the average of $R_{y}(t)$ of the chains at time $t$ under steady shear in the presence of the electric field (cf. Appendix B). This function as well as the end-to-end vector autocorrelation function in $y$ direction, $\Psi_{\mathrm{ss}}(t ; \dot{\gamma}) \equiv$ $\left\langle R_{y}(t) R_{y}(0)\right\rangle_{\text {ss }}$ defined in the absence of the electric field, can be calculated from eq 6 . For easy calcu- lation, we can expand the segment position $\mathbf{r}(n, t)$ with respect to the Rouse eigenfunctions, $\cos (p \pi n)$ $N$ ) with $p=0,1,2, \ldots$, as

$$
\mathbf{r}(n, t)=\sum_{p \geq 0}\left[\begin{array}{c}
X_{p}(t) \\
Y_{p}(t) \\
Z_{p}(t)
\end{array}\right] \cos \left(\frac{p \pi n}{N}\right)
$$

(The Rouse eigenfunctions are not necessarily the eigenfunctions associating $\boldsymbol{\Lambda}$ but are chosen as base functions for easy calculation.) $\Phi_{\mathrm{ss}}^{\mathrm{um}}(t ; \dot{\gamma})$ and $\Psi_{\mathrm{ss}}(t ; \dot{\gamma})$ are calculated with a standard method explained in Appendix B. The results are summarized as

$$
\Phi_{\mathrm{ss}}^{\mathrm{un}}(t ; \dot{\gamma})=\frac{2 \tilde{\mu}^{2} v}{N \zeta} \sum_{\beta \geq 1} g_{\beta}^{[\Phi]} \exp \left(-\lambda_{\beta} t\right)
$$

with

$$
\begin{aligned}
g_{\beta}^{[\Phi]}=\frac{1}{\lambda_{\beta}} & \left\{\sum_{p \geq 1}(\cos p \pi-1) Q_{3 p-1, \beta}\right\} \\
& \times\left\{\sum_{p^{\prime} \geq 1}\left(\cos p^{\prime} \pi-1\right) Q_{\beta, 3 p^{\prime}-1}^{-1}\right\}
\end{aligned}
$$

and

$$
\Psi_{\mathrm{ss}}(t ; \dot{\gamma})=\frac{2 k_{\mathrm{B}} T}{N \zeta} \sum_{\beta \geq 1} g_{\beta}^{[\Psi]} \exp \left(-\lambda_{\beta} t\right)
$$

with

$$
\begin{aligned}
g_{\beta}^{[\Psi]}=\sum_{\beta^{\prime} \geq 1} \frac{2}{\lambda_{\beta}+} \lambda_{\beta^{\prime}} & \left\{\sum_{\alpha \geq 1} Q_{\beta, \alpha}^{-1} Q_{\beta^{\prime}, \alpha}^{-1}\right\} \\
\times & \left\{\sum_{p \geq 1}(\cos p \pi-1) Q_{3 p-1, \beta}\right\} \\
\times & \left\{\sum_{p^{\prime} \geq 1}\left(\cos p^{\prime} \pi-1\right) Q_{\beta^{\prime}, 3 p^{\prime}-1}^{-1}\right\}
\end{aligned}
$$

The parameters $\lambda_{\beta}, Q_{i, j}$, and $Q_{i, j}^{-1}$ appearing in eqs 9 and 10, being dependent on $\dot{\gamma}$ in principle, are related to a matrix $\mathbf{A}$ with its components being defined by

$$
\begin{array}{lll}
A_{3 p-2,3 q-2}=a_{p, q}^{x x}, & A_{3 p-2,3 q-1}=a_{p, q}^{x y}+\zeta \dot{\gamma}, & A_{3 p-2,3 q}=a_{p, q}^{x z}, \\
A_{3 p-1,3 q-2}=a_{p, q}^{y x}, & A_{3 p-1,3 q-1}=a_{p, q}^{y y}, & A_{3 p-1,3 q}=a_{p, q}^{y z}, \\
A_{3 p, 3 q-2}=a_{p, q}^{z x}, & A_{3 p, 3 q-1}=a_{p, q}^{z y}, & A_{3 p, 3 q}=a_{p, q}^{z z} \quad(p, q=1,2, \ldots)
\end{array}
$$

Here, $a_{i, j}^{\alpha \beta}(i, j=1,2, \ldots ; \alpha, \beta=x, y, z)$ are the expansion coefficients of the Rouse eigenfunction defined with respect to the $\alpha \beta$ component of the operator, $\boldsymbol{\Lambda}_{\alpha \beta}$, as

Journal of Polymer Science: Part B: Polymer Physics DOI $10.1002 /$ polb 


$$
\begin{aligned}
\Lambda_{\alpha \beta}(n ; \dot{\gamma}) \cos \left(\frac{j \pi n}{N}\right) \\
\quad=\sum_{i \geq 1} a_{i, j}^{\alpha \beta} \cos \left(\frac{i \pi n}{N}\right)(i, j=1,2, . .)
\end{aligned}
$$

(Equivalently, $a_{i, j}^{\alpha \beta}=(2 / N) \int_{0}^{N} \mathrm{~d} n \cos (i \pi n / N)\left\{\Lambda_{\alpha \beta}\right.$ $\cos (j \pi n / N)\}$.) The parameter $\lambda_{\beta}$ is the $\beta$ th eigenvalue of the matrix $\mathbf{A}$ divided by $\zeta, Q_{i, j}$ is the $i, j$ component of a matrix $\mathbf{Q}$ that diagonalizes $\mathbf{A}$, and $Q_{i, j}^{-1}$ is the $i, j$ component of the inverse matrix $\mathbf{Q}^{-1}$; namely, $\left\{\mathbf{Q}^{-1} \mathbf{A} \mathbf{Q}\right\}_{\alpha \beta}=-\zeta \lambda_{\beta} \delta_{\alpha \beta}$ with $\delta_{\alpha \beta}$ being Kronecker delta.

\section{Comparison of $\Phi_{\mathrm{ss}}^{\mathrm{un}}(t ; \dot{\gamma})$ and $\Psi_{\mathrm{ss}}(t ; \dot{\gamma})$}

As seen from eqs $9 \mathrm{a}$ and $10 \mathrm{a}$, the relaxation times of the modes, $1 / \Lambda_{\beta}$, are exactly the same for the dielectric relaxation and end-to-end vector autocorrelation functions, $\Phi_{\mathrm{ss}}^{\mathrm{un}}$ and $\Psi_{\mathrm{ss}}$, calculated from the rather general Langevin equation (eq 6). Thus, even under steady shear, the terminal endto-end fluctuation time can be rigorously evaluated as the terminal dielectric relaxation time.

Now, we turn our attention to the mode distribution of $\Phi_{\mathrm{ss}}^{\mathrm{un}}$ and $\Psi_{\mathrm{ss}}$. The operator $\Lambda$ is determined by the effective Hamiltonian, and the coefficient $a_{p, q}^{\alpha \beta}$ appearing in eqs 11 and 12 is invariant on exchange of $\alpha$ and $\beta$, for example, $a_{p, q}^{x y}=a_{p, q}^{y x}$. Thus, at equilibrium, the matrix $\mathbf{A}$ is symmetric $\left(A_{i j}=A_{j i}\right.$ in eq 11 with $\left.\dot{\gamma} \rightarrow 0\right)$ and the diagonalizing matrix $\mathbf{Q}$ is orthogonal $\left(\mathbf{Q}^{-1}=\mathbf{Q}^{+}\right.$with $\mathbf{Q}^{+}=$ transpose matrix of $\mathbf{Q})$. Then, the term appearing in eq $10 \mathrm{~b}, \Sigma_{\alpha \geq 1} Q_{\beta, \alpha}^{-1} Q_{\beta^{\prime}, \alpha}^{-1}$, reduces to $\delta_{\beta \beta^{\prime}}$ so that the mode intensities $g_{\beta}^{[\Phi]}$ (eq 9b) and $g_{\beta}^{[\Psi]}$ (eq 10b) exactly coincide with each other. Since $\Phi^{\text {un }}$ and $\Psi$ have the same relaxation times $1 / \lambda_{\beta}$, this coincidence of $g$ means that $\Phi^{\mathrm{un}}$ and $\Psi$ are rigorously proportional to each other and satisfy a relationship $\Phi^{\mathrm{un}}(t)=\left\{\tilde{\mu}^{2} v / k_{\mathrm{B}} T\right\} \Psi(t)$ at any $t$. This fact confirms the validity of the Green-Kubo theorem at equilibrium.

Under steady shear, the symmetry of $\mathbf{A}$ breaks because of the $\zeta \dot{\gamma}$ term contributing to the component $A_{3 p-2,3 q-1}$ (cf. eq 11). If the other component $A_{3 p-1,3 q-2}$ has a nonzero value (which seems to be the case in general), the chain dynamics in $y$ direction is coupled with that in $x$ direction. For this case, $\Phi_{\mathrm{ss}}^{\mathrm{un}}$ and $\Psi_{\mathrm{ss}}$ are not rigorously proportional to each other. However, an argument based on the shear symmetry suggests an approximate proportionality, as explained below.
The relaxation rate $\Lambda_{\beta}$ as well as $\Phi_{\mathrm{ss}}^{\mathrm{un}}(t ; \dot{\gamma})$ and $\Psi_{\mathrm{ss}}(t ; \dot{\gamma})$ are invariant on inversion of the shear direction and thus even functions of $\dot{\gamma}$. Then, we can generally expand these quantities as

$$
\begin{gathered}
\lambda_{\beta}(\dot{\gamma})=\lambda_{\beta}^{(0)}+\dot{\gamma}^{2} \lambda_{\beta}^{(2)}+O\left(\dot{\gamma}^{4}\right) \\
\Phi_{\mathrm{ss}}^{\mathrm{un}}(t ; \dot{\gamma})=\Phi^{\mathrm{un}(0)}(t)+\dot{\gamma}^{2} \Phi_{\mathrm{ss}}^{\mathrm{un}(2)}(t)+O\left(\dot{\gamma}^{4} ; t\right) \\
\Psi_{\mathrm{ss}}(t ; \dot{\gamma})=\Psi^{(0)}(t)+\dot{\gamma}^{2} \Psi_{\mathrm{ss}}^{(2)}(t)+O\left(\dot{\gamma}^{4} ; t\right)
\end{gathered}
$$

where the superscript " $(0)$ " denotes the quantities at equilibrium $(\dot{\gamma} \rightarrow 0)$, the superscript "(2)" stands for the second-order expansion coefficients of respective quantities, and $O\left(\dot{\gamma}^{4}\right)$ and $O\left(\dot{\gamma}^{4} ; t\right)$ denote quantities of the order of $\dot{\gamma}^{4}$. Analyzing these coefficients on the basis of the shear symmetry, we can find the proportionality between $\Phi_{\mathrm{ss}}^{\mathrm{un}}(t ; \dot{\gamma})$, and $\Psi_{\mathrm{ss}}(t ; \dot{\gamma})$ (validity of the Green-Kubo theorem) at least up to the order of $\dot{\gamma}^{2}$, as explained in Appendix B. This validity, deduced from a "sufficient condition" for the odd and even symmetries of two types of correlation functions introduced in Appendix $\mathrm{B}$, seems to be a general consequence of the entanglement dynamics that is equivalent to the Rouse dynamics being modified due to topological constraints.

The validity of the Green-Kubo theorem discussed above, in particular the rigorous coincidence of the relaxation times of $\Phi_{\mathrm{ss}}^{\mathrm{un}}(t ; \dot{\gamma})$ and $\Psi_{\mathrm{ss}}(t$; $\dot{\gamma})$, was deduced from the Langevin analysis based on the linear operator $\Lambda$ (cf. eq 6). As explained in Appendix C, the other type of Langevin analysis without utilizing $\boldsymbol{\Lambda}$ also suggests the (approximate) validity, although this analysis cannot give explicit expressions of the relaxation times/intensities. Thus, the validity of the Green-Kubo theorem appears to be a rather general consequence of the Langevin analysis.

Apart from the above argument, we generally expect that $\Lambda_{\beta}, \Phi_{\mathrm{ss}}^{\mathrm{un}}$ and $\Psi_{\mathrm{ss}}$ are affected by the steady shear to the same order of $\dot{\gamma}$; compare eqs 13a-13c. Thus, in a semiquantitative sense, the shear effect should be insignificant for any two of $\Lambda_{\beta}, \Phi_{\mathrm{ss}}^{\mathrm{un}}, \Psi_{\mathrm{ss}}$ if the remaining one is not significantly affected by the shear in the range of $\dot{\gamma}$ examined. Experiments ${ }^{3,7}$ indicated that the terminal dielectric relaxation time $\tau_{\varepsilon}$ of entangled linear PI chains, being identical to the terminal time for the end-to-end vector fluctuation (cf. eqs $9 \mathrm{a}$ and 10a), is hardly affected by steady shear at rates $\dot{\gamma}<30 / \tau_{\varepsilon}^{0}$, with $\tau_{\varepsilon}^{0}$ being $\tau_{\varepsilon}$ at equilibrium. This fact means that the $\dot{\gamma}^{2}$-order term for $\lambda_{\beta}$ (eq 
13a) is actually negligible compared to the $\dot{\gamma}^{0}$ order term at those $\dot{\gamma}$. Thus, this should be the case also for $\Phi_{\mathrm{ss}}^{\mathrm{un}}$ (eq 13b) and $\Psi_{\mathrm{ss}}$ (eq 13c) of linear PI at $\dot{\gamma}<30 / \tau_{\varepsilon}^{0}$ and these $\Phi_{\mathrm{ss}}^{\mathrm{un}}$ and $\Psi_{\mathrm{ss}}$ should be satisfactorily approximated by the equilibrium functions obeying the Green-Kubo theorem.

All above results suggest that the Green-Kubo theorem is valid at least up to the order of $\dot{\gamma}^{2}$ and, more importantly, in the range of $\dot{\gamma}$ actually examined for PI. Consequently, the polarization $P_{\mathrm{ss}}(t)$ under steady shear can be satisfactorily expressed as

$P_{\mathrm{ss}}(t)=-\frac{v \tilde{\mu}^{2}}{k_{\mathrm{B}} T}\left\langle R_{y}^{2}\right\rangle_{\mathrm{ss}} \int_{-\infty}^{t} \mathrm{~d} s E(s) \frac{\partial \Phi_{\mathrm{ss}}(t-s)}{\partial t}$

(for entangled linear PI at $\dot{\gamma}<30 / \tau_{\varepsilon}^{0}$ )

with

$$
\Phi_{\mathrm{Ss}}(t)=\frac{\left\langle R_{\mathrm{y}}(t) R_{\mathrm{y}}(0)\right\rangle_{\mathrm{ss}}}{\left\langle R_{\mathrm{y}}^{2}\right\rangle_{\mathrm{ss}}}
$$

The corresponding dielectric intensity under steady shear, $\Delta \varepsilon_{\mathrm{ss}}$, is written as

$$
\Delta \varepsilon_{\mathrm{ss}}=F\left(\frac{4 \pi \tilde{\mu}^{2}}{k_{\mathrm{B}} T}\right) v\left\langle R_{y}^{2}\right\rangle_{\mathrm{ss}}
$$

where the correction factor for the internal electric field, $F$, and the prefactor of $4 \pi$ (in MKSA units) have been again introduced (cf. eq 4).

\section{Additional Comments}

Concerning the $\dot{\gamma}$-insensitivity of $\tau_{\varepsilon}\left(\cong 1 / \lambda_{1}\right)$ experimentally observed at $\dot{\gamma}<30 / \tau_{\varepsilon}^{0}$, one might argue that the chain motion at those $\dot{\gamma}$ remains essentially in equilibrium thereby giving $\lambda_{1} \cong \lambda_{1}{ }^{(\mathrm{eq})}$. However, this is not the case, as noted from significant nonlinearities of viscoelastic data observed in the same range of $\dot{\gamma}$. As an example, Fig. 1 shows the data of $\tau_{\varepsilon}, \Delta \varepsilon_{\mathrm{ss}}$, and the steady state viscosity $\eta$ measured for an entangled PI solution (molecular weight $M_{\mathrm{PI}}=1.2 \times 10^{6}$, concentration $C_{\mathrm{PI}}=15 \mathrm{wt} \%$ in an oligomeric solvent); see unfilled symbols. These data are normalized by the respective equilibrium values and plotted against the normalized shear rate $\dot{\gamma} \tau_{\varepsilon}^{0}$. The dielectric $\tau_{\varepsilon}$ and $\Delta \varepsilon_{\mathrm{ss}}$ are hardly dependent on $\dot{\gamma}$, while $\eta$ strongly decreases with increasing $\dot{\gamma}$. Thus, the PI chains at those $\dot{\gamma}$ exhibit prominently nonequilibrium dynamics that leads to the significant nonlinearity for the viscoelastically detected orientational anisotropy, together with a very small

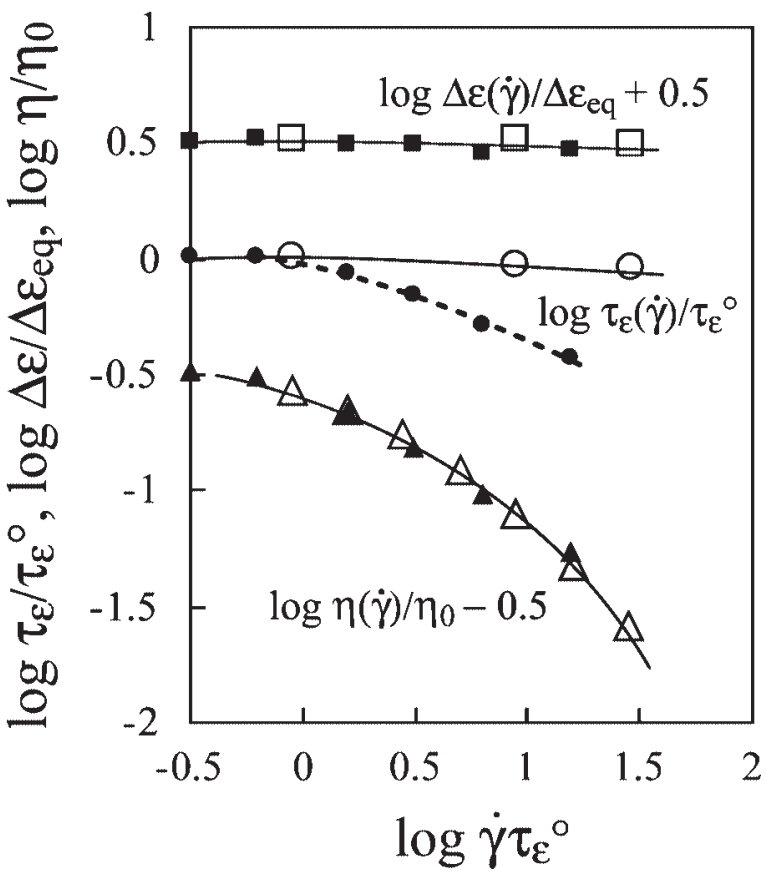

Figure 1. Dielectric relaxation time $\tau_{\varepsilon}(\dot{\gamma})$, dielectric relaxation intensity $\Delta \varepsilon(\dot{\gamma})$, and the viscosity $\eta(\dot{\gamma})$ measured at $30{ }^{\circ} \mathrm{C}$ for an entangled PI solution (in oligomeric butadiene; $M_{\mathrm{PI}}=1.2 \times 10^{6}$ and $C_{\mathrm{PI}}=15 \mathrm{wt} \%$ for PI) under fast steady shear. These data are normalized by respective zero-shear values and plotted against a reduced shear rate $\dot{\gamma} \tau_{\varepsilon}^{0}$; see unfilled symbols. The data were taken from ref. 7 , with permission. Filled symbols indicate the normalized $\tau_{\varepsilon}(\dot{\gamma}), \Delta \varepsilon(\dot{\gamma})$, and $\eta(\dot{\gamma})$ obtained from the NAPLES simulation (cf. Appendix D).

nonlinearity for the dielectrically detected end-toend fluctuation.

The shear-insensitivity of the rheodielectric data unequivocally indicates that the orientational memory of the end-to-end vector of the PI chains (in the shear gradient direction) is hardly affected by steady shear at $\dot{\gamma}<30 / \tau^{0}$. This fact could be related to a mechanism of flow-induced dynamic tube dilation for linear PI chains under steady shear. ${ }^{7}$ However, details of this mechanism have not been well addressed theoretically. In addition, no available molecular model appears to describe consistently/quantitatively the viscoelastic and rheodielectric data shown in Figure 1. Further theoretical study is strongly desired for this problem.

In relation to the difference between those viscoelastic and rheodielectric data, we remember that a molecular simulation (NAPLES simulation) based on the primitive chain network model ${ }^{25}$ reasonably mimicked the data. ${ }^{26}$ In the simulation, 
positive and negative charges $(\tilde{\mu}$ and $-\tilde{\mu}$ ) were placed at the chain ends, and the polarization due to a weak electric field was directly evaluated from the spatial distribution of the chain ends. (The resulting dielectric relaxation function was close to $\Phi_{\mathrm{ss}}(t)$ defined by eq $14 \mathrm{~b},{ }^{26}$ lending support to eq 14.) For obtaining better statistics of the NAPLES simulation, it was again made this study (cf. Appendix D) for a larger number of chains and over a longer time scale compared to the previous study. ${ }^{26}$ The simulated results, shown with the filled symbols in Figure 1, are considerably close to the data (unfilled symbols). Although the simulated $\tau_{\varepsilon}$ decreases with $\dot{\gamma}$ more rapidly than the experimental $\tau_{\varepsilon}$ data and the dynamics considered in the simulation needs to be refined, the simulation captures qualitatively a difference of the nonlinearities for $\tau_{\varepsilon}$ and $\eta$ (stronger for the latter).

\section{Dielectric Signal from PI under LAOS}

The dielectric response of entangled linear PI chains under LAOS has been examined experimentally. ${ }^{11,12}$ However, to our knowledge, no analysis has been made for the theoretical expression of the response under LAOS. We here attempt to make an approximate analysis of this response.

The validity of the Green-Kubo theorem under LAOS can be examined, in principle, through the Langevin analysis similar to that under the steady shear explained in the previous section. However, the analysis becomes much more complicated under LAOS because the shear rate tensor (cf. eq 7) oscillates with time for this case. Thus, considering the validity of the Green-Kubo theorem for PI under steady shear at $\dot{\gamma}<30 / \tau_{\varepsilon}^{0}$, we attempt to utilize eq 1 (representing this theorem) as a starting equation in our analysis under LAOS. For this attempt, we first need to examine a relationship between the dielectric $\tau_{\varepsilon}^{0}$ of PI at equilibrium and the maximum shear rate $\dot{\gamma}_{\max }=$ $\gamma_{0} \Omega$ for LAOS at the amplitude $\gamma_{0}$ and the angular frequency $\Omega$. The rheodielectric LAOS experiments ${ }^{10,11}$ were conducted under conditions, $\gamma_{0} \leq$ 1.6 and $\Omega \leq 3 / \tau_{\varepsilon}^{0}$ and $/$ or $\gamma_{0} \leq 3.6$ and $\Omega \cong 0.1 / \tau_{\varepsilon}^{\circ}$, that is, in a range of $\dot{\gamma}_{\max } \leq 5 / \tau_{\varepsilon}^{0}$. Thus, those experiments were conducted well in a range of $\dot{\gamma}$ $<30 / \tau_{\varepsilon}^{0}$ (where the Green-Kubo theorem holds under steady shear), satisfying the first criterion for the use of eq 1 under the LAOS conditions examined so far. As the second criterion, we need to examine if some fractional interval of time during one cycle of LAOS is long enough to realize a (pseudo) stationary state. Rigorously speaking, the stationary state is never realized under LAOS because the conformational distribution function should be synchronized with LAOS to oscillate at $\Omega$. However, the experimentally examined $\Omega$ is not significantly larger than $1 / \tau_{\varepsilon}^{0}$, which tempts us to approximate that the (pseudo) stationary state is realized in some fractional interval of time during one cycle of LAOS. This approximation is very crude but necessary for making a simple analysis of the rheodielectric response under LAOS. We believe that this crude approximation is acceptable as the first attempt of analysis in the absence of a full theory of this response.

Within this approximation, we utilize eq 1 as the starting equation in our LAOS analysis:

$$
\begin{aligned}
& P_{\mathrm{LAOS}}(t)=\frac{\tilde{\mu}^{2}}{k_{\mathrm{B}} T} \int_{-\infty}^{t} \mathrm{~d} s E(s) \\
& \times \int \mathrm{d} \mathbf{p} \mathrm{d} \mathbf{q}\left\{\left\{\Sigma_{j} R_{y, j}(t)\right\} \frac{\mathrm{d}}{\mathrm{d} s}\left\{\Sigma_{j} R_{y, j}(s)\right\}\right\} f_{\mathrm{LAOS}}(\mathbf{p}, \mathbf{q}, s)
\end{aligned}
$$

Here, $f_{\text {LAOS }}(\mathbf{p}, \mathbf{q}, s)$ is the distribution function in the phase space $(\mathbf{p}, \mathbf{q})$ at time $s$ under LAOS. ( $f_{\text {LAOS }}$ is defined in the absence of the electric field.) This $f_{\text {LAOS }}(\mathbf{p}, \mathbf{q}, s)$ is synchronized with LAOS to oscillate at $\Omega$ with some phase difference $\delta$ (defined with respect to the electric field at time 0 ). In the simplest case, $f_{\mathrm{LAOS}}(\mathbf{p}, \mathbf{q}, s)$ can be written in a form

$$
\begin{gathered}
f_{\mathrm{LAOS}}(\mathbf{p}, \mathbf{q}, s)=\frac{K^{\text {base }}(\mathbf{p}, \mathbf{q})+K^{\mathrm{osc}}(\mathbf{p}, \mathbf{q}) \sin (\Omega s+\delta)}{I^{\text {base }}+I^{\text {osc }} \sin (\Omega s+\delta)} \\
=\left\{\frac{K^{\text {base }}(\mathbf{p}, \mathbf{q})}{I^{\text {base }}}+\frac{K^{\text {osc }}(\mathbf{p}, \mathbf{q})}{I^{\text {osc }}} r \sin (\Omega s+\delta)\right\} \\
\times \sum_{\alpha \geq 0}(-r)^{\alpha} \sin ^{\alpha}(\Omega s+\delta) \quad(16)
\end{gathered}
$$

with

$$
\begin{aligned}
& I^{\text {base }}=\int \mathrm{d} \mathbf{p} \mathrm{d} \mathbf{q} K^{\mathrm{base}}(\mathbf{p}, \mathbf{q}), \\
& I^{\mathrm{osc}}=\int \mathrm{d} \mathbf{p} \mathrm{d} \mathbf{q} K^{\mathrm{osc}}(\mathbf{p}, \mathbf{q}), \text { and } \begin{aligned}
& r=\frac{I^{\text {osc }}}{I^{\text {base }}} \\
&(0<r<1)
\end{aligned}
\end{aligned}
$$

In eq $16, K^{\text {base }}(\mathbf{p}, \mathbf{q})$ and $K^{\mathrm{osc}}(\mathbf{p}, \mathbf{q})$ indicate the unnormalized distribution functions representing the nonoscillatory (base) and oscillatory parts of $f_{\text {LAOS }}(\mathbf{p}, \mathbf{q}, s)$, respectively. The denominator in 
the first line of eq 16 guarantees the normalization, $\int \mathrm{d} \mathbf{p} \mathrm{d} \mathbf{q} f_{\text {LAOS }}(\mathbf{p}, \mathbf{q}, s)=1$ at any $s$.

Substituting eq 16 into eq 15, we obtain

$$
\begin{aligned}
& P_{\text {LAOS }}(t)=\frac{v \tilde{\mu}^{2}}{k_{\mathrm{B}} T} \int_{-\infty}^{t} \mathrm{~d} s E(s)\left\{\frac{\partial \Psi^{\mathrm{base}}(t, s)}{\partial \mathrm{s}}\right. \\
& \left.+\frac{\partial \Psi^{\mathrm{osc}}(t, s)}{\partial \mathrm{s}} r \sin (\Omega s+\delta)\right\} \sum_{\alpha \geq 0}(-r)^{\alpha} \sin ^{\alpha}(\Omega s+\delta)
\end{aligned}
$$

where $v$ is the chain number density, and $\Psi^{\text {base }}(t, s)$ and $\Psi^{\mathrm{osc}}(t, s)$ are defined as

$$
\begin{aligned}
\Psi^{\text {base }}(t, s) & \equiv\left\langle R_{y}(t) R_{y}(s)\right\rangle_{\mathrm{LAOS}}^{\text {base }} \\
& =\frac{\int \mathrm{d} \mathbf{p} \mathrm{d} \mathbf{q}\left\{R_{y}(t) R_{y}(s)\right\} K^{\mathrm{base}}(\mathbf{p}, \mathbf{q})}{\int \mathrm{d} \mathbf{p} \mathrm{d} \mathbf{q} K^{\mathrm{base}}(\mathbf{p}, \mathbf{q})} \\
\Psi^{\mathrm{osc}}(t, s) & \equiv\left\langle R_{y}(t) R_{y}(s)\right\rangle_{\mathrm{LAOS}}^{\mathrm{osc}} \\
& =\frac{\int \mathrm{d} \mathbf{p} \mathrm{d} \mathbf{q}\left\{R_{y}(t) R_{y}(s)\right\} K^{\mathrm{osc}}(\mathbf{p}, \mathbf{q})}{\int \mathrm{d} \mathbf{p} \mathrm{d} \mathbf{q} K^{\mathrm{osc}}(\mathbf{p}, \mathbf{q})}
\end{aligned}
$$

As seen in eq 19, the polarization under LAOS, $P_{\mathrm{LAOS}}(t)$, includes information on the unnormalized correlation functions $\Psi^{\text {base }}(t, s)$ and $\Psi^{\text {osc }}(t, s)$. This information is analyzed below.

\section{Analysis of Components of $\boldsymbol{P}_{\mathrm{LAOS}}(\boldsymbol{t})$}

Rigorously speaking, the stationary state is never realized under LAOS because of the oscillation of $f_{\text {LAOS }}$, and both $\Psi^{\text {base }}(t, s)$ and $\Psi^{\text {osc }}(t, s)$ should depend on $t$ and $s$ separately. However, the correlation of the chain conformations at times $t$ and $s$, specified by $\Psi^{\text {base }}(t, s)$ and $\Psi^{\text {osc }}(t, s)$ (cf. eq 19), should decay as a function of the interval $t-s$ if this interval is sufficiently longer than the LAOS period. For this reason, we may approximate $\Psi^{\text {base }}(t, s)$ and $\Psi^{\text {osc }}(t, s)$ as functions of $t-s$ to examine the slow dynamics of the chain under LAOS. This approximation should lead to a smaller error compared to the approximate use of the Green-Kubo theorem (based on the assumption of pseudo stationary state in some fractional interval of time during one cycle of LAOS, as explained for eq 15) and is acceptable in our analysis based on this use.

From this argument, we may express $\Psi^{\text {base }}$ and $\Psi^{\text {osc }}$ as

$$
\begin{aligned}
\Psi^{\text {base }} \cong \Psi^{\text {base }}(t-s)=\left\langle R_{y}(t-s) R_{y}(0)\right\rangle_{\mathrm{LAOS}}^{\text {base }} \\
=\left\langle R_{y}^{2}\right\rangle_{\text {LAOS }}^{\text {base }} \sum_{\alpha \geq 1} g_{\text {base }, \alpha} \exp \left\{-\frac{(t-s)}{\tau_{\text {base }, \alpha}}\right\} \\
\Psi^{\text {osc }} \cong \Psi^{\text {osc }}(t-s)=\left\langle R_{y}(t-s) R_{y}(0)\right\rangle_{\mathrm{LAOS}}^{\text {osc }} \\
=\left\langle R_{y}^{2}\right\rangle_{\mathrm{LAOS}}^{\text {osc }} \sum_{\alpha \geq 1} g_{\text {osc }, \alpha} \exp \left\{-\frac{(t-s)}{\tau_{\text {osc }, \alpha}}\right\}
\end{aligned}
$$

with

$$
\sum_{\alpha \geq 1} g_{\text {base }, \alpha}=\sum_{\alpha \geq 1} g_{\text {osc }, \alpha}=1
$$

and

$$
\begin{aligned}
& \left\langle R_{y}^{2}\right\rangle_{\text {LAOS }}^{\text {base }}=\frac{\int \mathrm{d} \mathbf{p} \mathrm{d} \mathbf{q} R_{y}^{2} K^{\mathrm{base}}(\mathbf{p}, \mathbf{q})}{\int \mathrm{d} \mathbf{p} \mathrm{d} \mathbf{q} K^{\mathrm{base}}(\mathbf{p}, \mathbf{q})} \text { and } \\
& \left\langle R_{y}^{2}\right\rangle_{\mathrm{LAOS}}^{\mathrm{osc}}=\frac{\int \mathrm{d} \mathbf{p} \mathrm{d} \mathbf{q} R_{y}^{2} K^{\mathrm{osc}}(\mathbf{p}, \mathbf{q})}{\int \mathrm{d} \mathbf{p} \mathrm{d} \mathbf{q} K^{\mathrm{osc}}(\mathbf{p}, \mathbf{q})}
\end{aligned}
$$

In eq 20, we have factorized $\Psi^{\xi}(t-s)(\xi=$ base, osc) into an intensity part, $\left\langle R_{y}^{2}\right\rangle_{\mathrm{LAOS}}^{\xi}$, and a normalized, time-dependent part, $\Sigma_{\alpha \geq 1} g_{\xi, \alpha} \exp \{-(t-$ $\left.s) / \tau_{\xi, \alpha}\right\}(=1$ at $s=t)$. Substituting eq 20 into eq 18 with $E(s)=E_{0} \sin \omega s$ (small electric field), we find

$$
\begin{aligned}
\frac{k_{\mathrm{B}} T}{v \tilde{\mu}^{2} E_{0}} & P_{\mathrm{LAOS}}(t) \\
= & \left\langle R_{y}^{2}\right\rangle_{\mathrm{LAOS}}^{\text {base }}\left\{\left(\sum_{\alpha \geq 1} g_{\text {base }, \alpha} \frac{1}{1+\omega^{2} \tau_{\text {base }, \alpha}^{2}}\right) \sin \omega t\right. \\
& \left.-\left(\sum_{\alpha \geq 1} g_{\text {base }, \alpha} \frac{\omega \tau_{\text {base }, \alpha}}{1+\omega^{2} \tau_{\text {base }, \alpha}^{2}}\right) \cos \omega t\right\} \\
& -\left\langle R_{y}^{2}\right\rangle_{\text {LAOS }}^{\text {base }} \frac{r}{2}\left\{S_{-}^{\text {base }}(\omega, \Omega) \sin (\omega-\Omega) t\right. \\
& \left.+C_{-}^{\text {base }}(\omega, \Omega) \cos (\omega-\Omega) t\right\} \\
& +\left\langle R_{y}^{2}\right\rangle_{\text {LAOS }}^{\text {base }} \frac{r}{2}\left\{S_{+}^{\text {base }}(\omega, \Omega) \sin (\omega+\Omega) t\right. \\
& \left.+C_{+}^{\text {base }}(\omega, \Omega) \cos (\omega+\Omega) t\right\} \\
& +\left\langle R_{y}^{2}\right\rangle_{\text {LAOS }} \frac{r}{2}\left\{S_{-}^{\text {osc }}(\omega, \Omega) \sin (\omega-\Omega) t\right. \\
& \left.+C_{-}^{\text {osc }}(\omega, \Omega) \cos (\omega-\Omega) t\right\} \\
& -\left\langle R_{y}^{2}\right\rangle_{\text {LAOS }}^{\text {osc }} \frac{r}{2}\left\{S_{+}^{\text {osc }}(\omega, \Omega) \sin (\omega+\Omega) t\right. \\
& \left.+C_{+}^{\text {osc }}(\omega, \Omega) \cos (\omega+\Omega) t\right\} \\
& +O\left(r^{\beta} \sin (\omega \pm \beta \Omega) ; \beta \geq 2\right)
\end{aligned}
$$

with

Journal of Polymer Science: Part B: Polymer Physics DOI $10.1002 /$ polb 


$$
\begin{aligned}
& S_{-}^{\xi}(\omega, \Omega)=\sum_{\alpha \geq 1} g_{\xi, \alpha} \frac{(\omega-\Omega) \tau_{\xi, \alpha} \cos \delta+\sin \delta}{1+(\omega-\Omega)^{2} \tau_{\xi, \alpha}^{2}}, \\
& C_{-}^{\xi}(\omega, \Omega)=\sum_{\alpha \geq 1} g_{\xi, \alpha} \frac{\cos \delta-(\omega-\Omega) \tau_{\xi, \alpha} \sin \delta}{1+(\omega-\Omega)^{2} \tau_{\xi, \alpha}^{2}}, \\
& S_{+}^{\xi}(\omega, \Omega)= \sum_{\alpha \geq 1} g_{\xi, \alpha} \frac{(\omega+\Omega) \tau_{\xi, \alpha} \cos \delta-\sin \delta}{1+(\omega+\Omega)^{2} \tau_{\xi, \alpha}^{2}}, \\
& C_{+}^{\xi}(\omega, \Omega)= \sum_{\alpha \geq 1} g_{\xi, \alpha} \frac{\cos \delta+(\omega+\Omega) \tau_{\xi, \alpha} \sin \delta}{1+(\omega+\Omega)^{2} \tau_{\xi, \alpha}^{2}} \\
&(\xi=\text { base }, \text { osc })
\end{aligned}
$$

$P_{\mathrm{LAOS}}(t)$ splits into a series of components oscillating at the angular frequency of the electric field $\omega$ and at $\omega \pm \beta \Omega$ with $\beta=1,2, \ldots$, as clearly seen in eq 23. The components oscillating at $\omega \pm \beta \Omega$ emerge because $P_{\mathrm{LAOS}}(t)$ detects the oscillation of $f_{\text {LAOS }}$ due to LAOS being coupled with the oscillatory electric field. This coupling makes a contrast between the rheodielectric responses under LAOS and steady shear: The response under steady shear oscillates only at $\omega$ (and is more easily analyzed compared to that under LAOS) because the distribution function under steady shear is independent of time.

The information for the correlation function $\Psi^{\text {base }}(t)$ under LAOS, that is, the mode distribution $\left\{g_{\text {base }, \alpha}, \tau_{\text {base }, \alpha}\right\}$ and the intensity $\left\langle R_{y}^{2}\right\}_{\text {LAOS }}^{\text {base }}$ (eqs $20 \mathrm{a}$ and 22), is included in the component of $P_{\mathrm{LAOS}}(t)$ oscillating at $\omega$; see the first term in eq 23 . On the other hand, the information for $\Psi^{\text {osc }}(t)$, $\left\{g_{\text {osc }, \alpha}, \tau_{\text {osc }, \alpha}\right\}$ and $\left\langle R_{y}^{2}\right\rangle_{\text {LAOS }}^{\text {osc }}$ (eqs $20 \mathrm{~b}$ and 22 ), is included in the components oscillating at $\omega \pm \beta \Omega$ $(\beta=1,2, \ldots)$; see the coefficients $S$ and $C$ given by eq 24. Namely, the full information for the chain dynamics under LAOS, being represented by a set of $\Psi^{\text {base }}(t)$ and $\Psi^{\mathrm{osc}}(t)$, can be obtained only when all these components of $P_{\mathrm{LAOS}}(t)$ are measured. However, in the rheodielectric studies under LAOS conducted so far, ${ }^{11,12}$ only the component oscillating at $\omega$ was measured and converted to $\varepsilon_{\text {LAOS }}^{\prime}(\omega)$ and $\varepsilon_{\text {LAOS }}^{\prime \prime}(\omega)$. Thus, the full information for the chain dynamics under LAOS was not obtained in those studies.

Nevertheless, it is still informative to examine the molecular meaning of the $\varepsilon_{\mathrm{LAOS}}^{\prime \prime}(\omega)$ data in literature. ${ }^{11,12}$ From the second term in the first line of eq 23 , we find

$$
\varepsilon_{\text {LAOS }}^{\prime \prime}(\omega)=\Delta \varepsilon_{\text {LAOS }} \sum_{\alpha \geq 1} g_{\text {base }, \alpha} \frac{\omega \tau_{\text {base }, \alpha}}{1+\omega^{2} \tau_{\text {base }, \alpha}^{2}}
$$

with

Journal of Polymer Science: Part B: Polymer Physics DOI $10.1002 /$ polb

$$
\Delta \varepsilon_{\mathrm{LAOS}}=F\left(\frac{4 \pi \tilde{\mu}^{2}}{k_{\mathrm{B}} T}\right) v\left\langle R_{y}^{2}\right\rangle_{\mathrm{LAOS}}^{\text {base }}
$$

(We have again introduced the correction factor $F$ for the internal electric field and the prefactor of $4 \pi$ in MKSA units; cf. eq 4). For well entangled linear PI, Höfl et al. ${ }^{11}$ and Capaccioli et al. ${ }^{12}$ showed that the $\omega$ dependence of the $\varepsilon_{\text {LAOS }}^{\prime \prime}(\omega)$ data (i.e., relative dielectric mode distribution) hardly changes under LAOS, in particular at low $\omega$ where the global dynamics of PI is detected. This fact suggests that the relaxation time and mode distribution of the unnormalized correlation function, $\Psi^{\text {base }}(t)$, are insensitive to LAOS. This result appears to be in harmony with the shearrate insensitivity of the rheodielectric data of PI measured under steady shear. ${ }^{7}$

\section{Further Examination of $\varepsilon^{\prime \prime}{ }_{\text {LAOS }}(\omega)$ Data}

For well-entangled bulk PI $\left(M_{\mathrm{PI}}=55 \times 10^{3} ; \sim 10\right.$ entanglements per chain), Höfl et al. ${ }^{11}$ reported that the rheodielectric intensity $\Delta \varepsilon_{\mathrm{LAOS}}$ (evaluated from the $\varepsilon_{\mathrm{LAOS}}^{\prime \prime}(\omega)$ data) decreased rather significantly with increasing LAOS amplitude $\gamma_{0}$. Specifically, at the LAOS frequency $\Omega \cong 3 / \tau_{\varepsilon}^{0}$, $\Delta \varepsilon_{\text {LAOS }}$ decreased by a factor of $\cong 30 \%$ with increasing $\gamma_{0}$ to $1.6 .{ }^{11}$ (A decrease of $\Delta \varepsilon_{\mathrm{LAOS}}$, though weaker in magnitude, was also reported by Capaccioli et al. ${ }^{12}$ ).

On the basis of eq 26, one may attribute this decrease of $\Delta \varepsilon_{\mathrm{LAOS}}$ to a decrease of the average size of a PI chain in the shear gradient direction. However, this assignment is not valid because the squared average of the chain size, $\left\langle R_{y}^{2}\right\rangle_{\mathrm{LAOS}}$, is correctly defined as an average of $R_{y}^{2}$ over the distribution function $f_{\text {LAOS }}(\mathbf{p}, \mathbf{q}, s)$ during one cycle of LAOS (for a time period of $2 \pi / \Omega$ ), not as the average $\left\langle R_{y}^{2}\right\rangle_{\text {LAOS }}^{\text {base }}$ defined with respect to the nonoscillatory part of this function, $K^{\text {base }}(\mathbf{p}, \mathbf{q})$ (cf. eq 22). From the first line in eq 16 together with a mathematical formula, $\int_{0}^{\pi} \mathrm{d} x\{1+\alpha \cos x\}^{-1}=\pi / \sqrt{1-\alpha^{2}}$ $(0<\alpha<1)$, we find

$$
\begin{aligned}
&\left\langle R_{y}^{2}\right\rangle_{\mathrm{LAOS}} \equiv \frac{\Omega}{2 \pi} \int_{0}^{2 \pi / \Omega} \mathrm{d} s \int \mathrm{d} \mathbf{p} \mathrm{d} \mathbf{q} R_{y}^{2} f_{\mathrm{LAOS}}(\mathbf{p}, \mathbf{q}, s) \\
&=\left\langle R_{y}^{2}\right\rangle_{\mathrm{LAOS}}^{\text {base }}\left\{\frac{1-\theta}{\sqrt{1-r^{2}}}\right\}+\left\langle R_{y}^{2}\right\rangle_{\mathrm{LAOS}}^{\text {osc }}
\end{aligned}
$$

with 


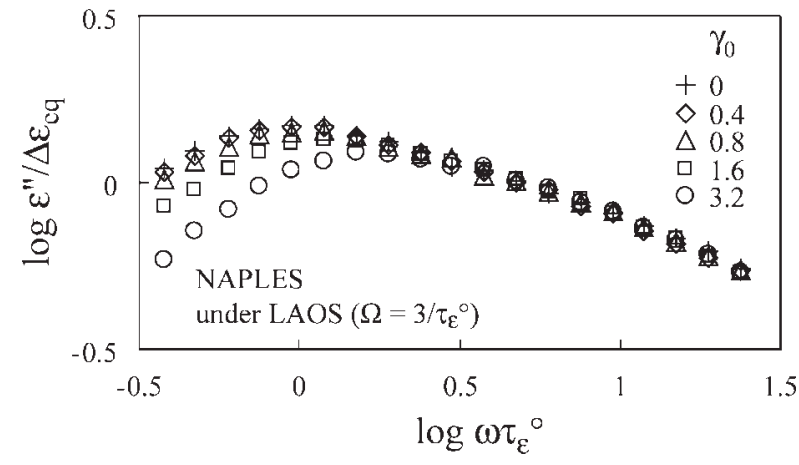

Figure 2. Dielectric loss $\varepsilon^{\prime \prime}$ LAOS $(\omega)$ of entangled PI (9.6 entanglements per chain on average) under LAOS obtained from the NAPLES simulation; see Appendix D. The LAOS condition, $\gamma_{0}(\leq 3.2)$ and $\Omega(=3 /$ $\left.\tau_{\varepsilon}^{0}\right)$, is similar to the experimental condition utilized by Höfl et al. ${ }^{11} \varepsilon^{\prime \prime}$ LAOS $(\omega)$ is normalized by the dielectric intensity at equilibrium, $\Delta \varepsilon_{\mathrm{eq}}$, and plotted against normalized frequency of the electric field, $\omega \tau_{\varepsilon}^{0}$.

$$
\theta=\frac{\left\langle R_{y}^{2}\right\rangle_{\mathrm{LAOS}}^{\mathrm{osc}}}{\left\langle R_{y}^{2}\right\rangle_{\mathrm{LAOS}}^{\text {base }}} \text { and } r=\frac{I^{\mathrm{osc}}}{I^{\text {base }}}(0<r<1)
$$

In general, the oscillatory part of the distribution function, $K^{\text {osc }}(\mathbf{p}, \mathbf{q})$, would give a stronger rheodielectric effect compared to the base part, $K^{\text {base }}(\mathbf{p}, \mathbf{q})$, and thus $r\left(=I^{\text {osc }} / I^{\text {base }}=\left\{\int \mathrm{d} \mathbf{p} \mathrm{d} \mathbf{q}\right.\right.$ $\left.\left.\times K^{\text {osc }}(\mathbf{p}, \mathbf{q})\right\} /\left\{\int \operatorname{dpd} \mathbf{q} K^{\text {base }}(\mathbf{p}, \mathbf{q})\right\}\right)$ would increase with increasing $\gamma_{0}$. On this increase of $r,\left\langle R_{y}^{2}\right\rangle_{\text {LAOS }}$ would become considerably larger than $\left\langle R_{y}^{2}\right\rangle_{\mathrm{LAOS}}^{\text {base }}$ (as can be noted from eq 27a), suggesting that the decrease of $\Delta \varepsilon_{\mathrm{LAOS}}\left(\propto\left\langle R_{y}^{2}\right\rangle_{\mathrm{LAOS}} \mathrm{Lase}_{\mathrm{LOS}}\right)$ is not equivalent to a decrease of $\left\langle R_{y}^{2}\right\rangle_{\text {LAOS }}$ by the same magnitude. We also note that the rheodielectric intensity under steady shear, $\Delta \varepsilon_{\mathrm{ss}} \sim\left\langle R_{y}^{2}\right\rangle_{\mathrm{ss}}$ (cf. eq 14c), hardly decreases on an increase of shear rate up to $30 / \tau_{\varepsilon}^{0}$; see Figure 1 . This fact suggests that the PI chain dimension in the shear gradient direction is not significantly affected by the fast steady shear (and possibly not by LAOS either).

The above difference between $\left\langle R_{y}^{2}\right\rangle_{\mathrm{LAOS}}^{\text {base }}$ $\left(\sim \Delta \varepsilon_{\text {LAOS }}\right)$ and $\left\langle R_{y}^{2}\right\rangle_{\text {LAOS }}$ can be further examined from the NAPLES simulation. For this purpose, we conducted the simulation for type-A chains (9.6 entanglements per chain on average) under the LAOS condition, as explained in Appendix D. The LAOS frequency, $\Omega$, was set at $3 / \tau_{\varepsilon}^{0}$, which mimics the experimental condition by Höfl et al. ${ }^{11}$ Each chain has small charges $\tilde{\mu}$ and $-\tilde{\mu}$ at its ends, as explained in the previous section. In the simulation, the macroscopic polarization and the corresponding $\varepsilon_{\text {LAOS }}^{\prime \prime}(\omega)$ were directly evaluated from the spatial distribution of the chain ends without utilizing any correlation function. In Figure 2, the simulated $\varepsilon_{\mathrm{LAOS}}^{\prime \prime}(\omega)$ is normalized by the dielectric intensity $\Delta \varepsilon_{\mathrm{eq}}$ at equilibrium and plotted double-logarithmically against the normalized frequency of the electric field, $\omega \tau_{\varepsilon}^{0}$. The simulated dielectric relaxation time $\tau_{\varepsilon}\left(\gamma_{0} ; \Omega\right)$ ( $\sim$ reciprocal of the peak frequency) decreases with increasing $\gamma_{0}$. Experiments indicated no significant decrease of $\tau_{\varepsilon}\left(\gamma_{0} ; \Omega\right)$ with $\gamma_{0},{ }^{11,12}$ which suggests a need to refine the chain dynamics considered in the simulation. However, the simulated dielectric mode distribution (seen as the shape of the $\varepsilon_{\text {LAOS }}^{\prime \prime}$ curve) is not significantly affected by LAOS, which is in harmony with the experiments. ${ }^{11,12}$

In the simulation, $\left\langle R_{y}^{2}\right\rangle_{\text {LAOS }}$ was evaluated straightforwardly as the squared end-to-end distance of the chain in the shear gradient direction averaged over many chains during one cycle of LAOS, and the $\left\langle R_{y}^{2}\right\rangle_{\text {LAOS }} /\left\langle R_{y}^{2}\right\rangle_{\text {eq }}$ ratio was obtained consequently. The dielectric intensity $\Delta \varepsilon_{\text {LAOS }}$ was obtained from the simulated $\varepsilon_{\text {LAOS }}^{\prime \prime}$ (Fig. 2), and the $\left\langle R_{y}^{2}\right\rangle_{\mathrm{LAOS}}^{\text {base }} /\left\langle R_{y}^{2}\right\rangle_{\text {eq }}$ ratio was evaluated as a ratio of $\Delta \varepsilon_{\mathrm{LAOS}}$ under LAOS to $\Delta \varepsilon_{\mathrm{eq}}$ at equilibrium. In

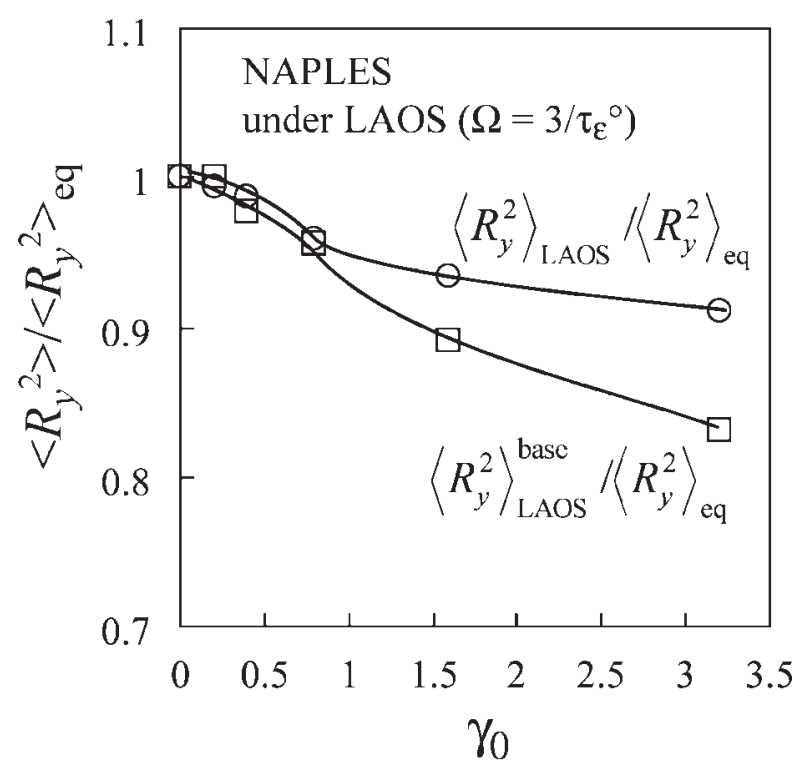

Figure 3. $\left\langle R_{y}^{2}\right\rangle_{\mathrm{LAOS}} /\left\langle R_{y}^{2}\right\rangle_{\mathrm{eq}}$ and $\left\langle R_{y}^{2}\right\rangle_{\mathrm{LAOS}}^{\mathrm{base}} /\left\langle R_{y}^{2}\right\rangle_{\mathrm{eq}}$ ratios obtained from the NAPLES simulation for entangled PI (9.6 entanglements per chain on average) under LAOS; see Appendix D. The LAOS condition, $\gamma_{0}(\leq$ 3.2) and $\Omega\left(=3 / \tau_{\varepsilon}^{0}\right)$, is similar to the experimental condition utilized by Höfl et al. ${ }^{11}$ The ratios are plotted against the LAOS amplitude, $\gamma_{0}$.

Journal of Polymer Science: Part B: Polymer Physics DOI 10.1002/polb 
Figure 3 , the $\left\langle R_{y}^{2}\right\rangle_{\mathrm{LAOS}} /\left\langle R_{y}^{2}\right\rangle_{\text {eq }}$ and $\left\langle R_{y}^{2}\right\rangle_{\mathrm{LAOS}}^{\mathrm{b}_{\mathrm{Lase}}} /\left\langle R_{y}^{2}\right\rangle_{\mathrm{eq}}$ ratios thus obtained are plotted against the LAOS amplitude, $\gamma_{0}$. Clearly, the $\left\langle R_{y}^{2}\right\rangle_{\mathrm{LAOS}}^{\text {base }} /\left\langle R_{y}^{2}\right\rangle_{\text {eq }}$ ratio (squares) decreases considerably with increasing $\gamma_{0}$ to 3.2 (which mimics the experiments ${ }^{11}$ ) while the $\left\langle R_{y}^{2}\right\rangle_{\text {LAOS }} /\left\langle R_{y}^{2}\right\rangle_{\text {eq }}$ ratio (circles) decreases less significantly. This result is in harmony with the above discussion for difference between $\left\langle R_{y}^{2}\right\rangle_{\mathrm{LAOS}}^{\text {base }}$ and $\left\langle R_{y}^{2}\right\rangle_{\text {LAOS }}$

\section{CONCLUDING REMARKS}

We have examined the molecular expression of rheodielectric response of type-A chains (e.g., PI) having small dipoles. The Langevin analysis suggested that the relaxation time is exactly the same for the rheodielectric relaxation function and the end-to-end vector autocorrelation function under steady shear and that the relaxation mode distribution coincides for these functions at least up to the second order of shear rate. Thus, the GreenKubo theorem satisfactorily describes the rheodielectric response under steady shear up to a fairly high shear rate where the rheological nonlinearity is clearly observed experimentally (while the rheodielectric data are not much affected by the shear). Consequently, the rheodielectric intensity at those shear rates is proportional to the meansquare chain size in the shear gradient direction, $\left\langle R_{y}^{2}\right\rangle$ (= average over the time-independent conformational distribution function).

In contrast, under LAOS, the distribution function $f_{\text {LAOS }}$ is synchronized with LAOS to split into a time-independent base part and an oscillatory part. Correspondingly, the rheodielectric response (polarization $P_{\mathrm{LAOS}}(t)$ ) also splits into components oscillating at $\omega$ and $\omega \pm \beta \Omega(\beta=1,2, \ldots)$, where $\omega$ and $\Omega$ are the angular frequencies of electric field and LAOS, respectively. The components of $P_{\text {LAOS }}(t)$ oscillating at $\omega$ and $\omega \pm \beta \Omega$ correspond to the end-to-end vector correlation averaged over the base and oscillatory parts of $f_{\mathrm{LAOS}}$, respectively. Because of the splitting, the $\varepsilon_{\mathrm{LAOS}}^{\prime \prime}(\omega)$ data (equivalent to the component of $P_{\text {LAOS }}$ oscillating at $\omega$ ) do not have the intensity being proportional to $\left\langle R_{y}^{2}\right\rangle$. In other words, the $\varepsilon_{\mathrm{LAOS}}^{\prime \prime}(\omega)$ data cannot give the information for the mean-square chain size defined with respect to $f_{\text {LAOS }}$. However, the $\varepsilon_{\text {LAOS }}^{\prime \prime}(\omega)$ data can still give some useful information for the chain dynamics: the relative mode distribution and relaxation time of the data are equivalent to those of the end-to-end vector fluctuation averaged over the base part of $f_{\mathrm{LAOS}}$. Thus, the experimentally observed LAOS insensitivity of the relaxation time of the $\varepsilon_{\mathrm{LAOS}}^{\prime \prime}(\omega)$ data suggests the LAOS insensitivity of the fluctuation time defined with respect to this part of $f_{\mathrm{LAOS}}$.

This work was partly supported by Grant-in-Aid for Scientific Research on Priority Area "Soft Matter Physics" from the Ministry of Education, Culture, Sports, Science and Technology, Japan (grant \#18068009). $\mathrm{K}$. Horio is supported by Research Fellowship for Young Scientists from Japan Society for the Promotion of Science. J. A. Pathak and C. M. Roland are supported by the Office of Naval Research.

\section{APPENDIX A: DERIVATION OF EQ 1 AT EQUILIBRIUM ${ }^{13}$}

Applying a time $(t)$ dependent weak electric field $E(t)$ to an equilibrium system of type-A chains, we measure the macroscopic polarization $P(t)$ in the direction of the field ( $=y$ direction). This $P(t)$ is expressed in terms of the end-to-end dipole $\tilde{\mu} R_{y, j}$ of $j$ th chain in the system (with $\tilde{\mu}=$ type-A dipole moment per unit length of the chain backbone and $R_{y, j}=y$ component of the end-to-end vector) as $P(t)=\int \mathrm{d} \mathbf{p} \operatorname{dq}\left\{\Sigma_{j} \tilde{\mu} R_{y, j}\right\}$ $f(\mathbf{p}, \mathbf{q}, t)$. Here, $f(\mathbf{p}, \mathbf{q}, t)$ is the conformational distribution function in the presence of the electric field: $f(\mathbf{p}, \mathbf{q}, t)$ is defined in the phase space specified by general momentum $\mathbf{p}$ and coordinate $\mathbf{q}$. For the weak field $E(t), f(\mathbf{p}, \mathbf{q}, t)$ is given by a sum of the time-independent equilibrium distribution function, $f^{\circ}(\mathbf{p}, \mathbf{q})$, and a perturbation due to the small electric field, $\Delta f(\mathbf{p}, \mathbf{q}, t)$. Since the macroscopic polarization is exclusively due to the electric field (i.e., $\int \mathrm{d} \mathbf{p} \mathrm{d} \mathbf{q}\left\{\Sigma_{j} \tilde{\mu} R_{y, j}\right\}$ $\left.f^{o}(\mathbf{p}, \mathbf{q})=0\right), P(t)$ is written as

$$
P(t)=\int \mathrm{d} \mathbf{p} \mathrm{d} \mathbf{q}\left\{\Sigma_{j} \tilde{\mu} R_{y, j}\right\} \Delta f(\mathbf{p}, \mathbf{q}, t)
$$

The Hamiltonian of the system, determining the time evolution of $\Delta f(\mathbf{p}, \mathbf{q}, t)$ and thus of $P(t)$, is given by

$$
\mathcal{H}(\mathbf{p}, \mathbf{q}, t)=\mathcal{H}^{0}(\mathbf{p}, \mathbf{q})-\left\{\Sigma_{j} \tilde{\mu} R_{y_{j}}\right\} E(t)
$$

Here, $\mathcal{H}^{\circ}(\mathbf{p}, \mathbf{q})$ is the equilibrium Hamiltonian for the configuration $(\mathbf{p}, \mathbf{q})$ in the phase space, and the $-\left\{\Sigma_{j} \tilde{\mu} R_{y, j}\right\} E(t)$ term represents the electrostatic energy under the electric field. The time evolution of the distribution function $f\left(=f^{\circ}+\Delta f\right)$ is described by an equation $\partial f / \partial t=[\mathcal{H}, f]$, where $[A, B]$ is the Poisson bracket defined as $[A, B]$ $\equiv(\partial A / \partial \mathbf{q})(\partial B / \partial \mathbf{p})-(\partial A / \partial \mathbf{p})(\partial B / \partial \mathbf{q})$ for physical 
quantities $A(\mathbf{p}, \mathbf{q})$ and $B(\mathbf{p}, \mathbf{q}) \cdot{ }^{14,15}$ Since $f^{0}$ is independent of time (i.e., $\partial f^{\circ} / \partial t=\left[\mathcal{H}^{\circ}, f^{\circ}\right]=0$ ) and the perturbation due to the electric field is small (i.e., $\left[\left\{\Sigma_{j} \tilde{\mu} R_{y, j}\right\} E, \Delta f\right] \cong 0$ ), the time evolution equation of $\Delta f$ can be expressed as

$$
\begin{aligned}
\frac{\partial \Delta f}{\partial t} & =\left[\mathcal{H}^{\mathrm{o}}, \Delta f\right]-\left[\left\{\Sigma_{j} \tilde{\mu} R_{y_{j}}\right\}, f^{\mathrm{o}}\right] E(t) \\
& =\left[\mathcal{H}^{\mathrm{o}}, \Delta f\right]+\left[\left\{\Sigma_{j} \tilde{\mu} R_{y_{j}}\right\}, \mathcal{H}^{\mathrm{o}}\right] \frac{f^{\mathrm{o}}(\mathbf{p}, \mathbf{q})}{k_{\mathrm{B}} T} E(t)
\end{aligned}
$$

In eq $\mathrm{A} 3$, we have utilized a relationship, $f^{\circ} \sim$ $\exp \left(-\mathcal{H}^{\circ} / k_{\mathrm{B}} T\right)$ with $k_{\mathrm{B}}=$ Boltzmann constant and $T=$ absolute temperature. Equation A3 can be readily solved to give

$$
\begin{array}{r}
\Delta f(\mathbf{p}, \mathbf{q}, t)=\int_{-\infty}^{t} \mathrm{~d} s E(s) e^{-(t-s) \mathcal{L}}\left[\left\{\Sigma_{j} \tilde{\mu} R_{y, j}\right\}, \mathcal{H}^{\mathrm{o}}\right] \\
\times \frac{f^{\mathrm{o}}(\mathbf{p}, \mathbf{q})}{k_{\mathrm{B}} T}
\end{array}
$$

where $\mathcal{L}$ is the Liouville operator defined as $\mathcal{L} A$ $\equiv\left[\mathcal{H}^{\mathrm{o}}, A\right]$.

Substituting eq A4 into eq A1, we find

$$
\begin{aligned}
& P(t)=\int_{-\infty}^{t} \mathrm{~d} s E(s) \int \mathrm{d} \mathbf{p} \mathrm{d} \mathbf{q}\left\{\Sigma_{j} \tilde{\mu} R_{y_{j}}\right\} e^{-(t-s) \mathcal{L}} \\
& \times\left[\left\{\Sigma_{j} \tilde{\mu} R_{y_{j}}\right\}, \quad \mathcal{H}^{0}\right] \frac{f^{\circ}(\mathbf{p}, \mathbf{q})}{k_{\mathrm{B}} T}=\int_{-\infty}^{t} \mathrm{~d} s E(s) \int \mathrm{d} \mathbf{p} \mathrm{d} \mathbf{q} \\
& \times\left\{e^{(t-s) \mathcal{L}}\left\{\Sigma_{j} \tilde{\mu} R_{y_{j}}\right\}\right\}\left[\left\{\Sigma_{j} \tilde{\mu} R_{y_{j}}\right\}, \mathcal{H}^{\circ}\right] \frac{f^{\mathrm{o}}(\mathbf{p}, \mathbf{q})}{k_{\mathrm{B}} T}
\end{aligned}
$$

In eq $\mathrm{A} 5$, we have utilized a feature of the Liouville operator, $\int \mathrm{d} \mathbf{p} \mathrm{d} \mathbf{q} A(\mathbf{p}, \mathbf{q})\left\{\mathcal{L}^{n} B(\mathbf{p}, \mathbf{q})\right\}=\int \mathrm{d} \mathbf{p}$ $\operatorname{dq}\left\{(-\mathcal{L})^{n} A(\mathbf{p}, \mathbf{q})\right\} B(\mathbf{p}, \mathbf{q})$. The terms $\left\{\Sigma_{j} \tilde{\mu} R_{y, j}\right\}$ and $\left[\left\{\Sigma_{j} \tilde{\mu} R_{y, j}\right\}, \mathcal{H}^{\circ}\right]$ appearing in eq A5 are defined at time $s$. These terms are rewritten as $\left[\left\{\Sigma_{j} \tilde{\mu} R_{y, j}\right\}\right.$, $\left.\mathcal{H}^{\circ}\right]=-\left[\mathcal{H}^{\mathrm{o}},\left\{\Sigma_{j} \quad \tilde{\mu} R_{y_{j}}\right\}\right]=\mathrm{d}\left\{\Sigma_{j} \quad \tilde{\mu} R_{y_{j}}(s)\right\} / \mathrm{d} s$ and $e^{(t-s) \mathcal{L}}\left\{\Sigma_{j} \tilde{\mu} R_{y_{j}}\right\}_{\text {at time } s}=\left\{\Sigma_{j} \tilde{\mu} R_{y_{j}}\right\}_{\text {at time }}$, where we have utilized features of the Poisson bracket and Liouville operator, $\left[\mathcal{H}^{\mathrm{o}}, A\right]_{\text {at time } s}=-\mathrm{d} A / \mathrm{d} s$ and $e^{t \mathcal{L}} A_{\text {at time } 0}=A_{\text {at time } t}$ for a physical quantity $A$. Considering these results, we can rewrite eq A5 as

$$
\begin{array}{r}
P(t)=\frac{\tilde{\mu}^{2}}{k_{\mathrm{B}} T} \int_{-\infty}^{t} \mathrm{~d} s E(s) \int \mathrm{d} \mathbf{p} \mathrm{d} \mathbf{q}\left\{\Sigma_{j} R_{y, j}(t)\right\} \\
\times \frac{\mathrm{d}}{\mathrm{d} s}\left\{\Sigma_{j} R_{y, j}(s)\right\} f^{\mathrm{o}}(\mathbf{p}, \mathbf{q})
\end{array}
$$

Equation A6 is identical to eq 1 in the text.

\section{APPENDIX B: LANGEVIN ANALYSIS}

\section{Solution of Langevin Equation}

The Langevin equation describing the time evolution of the segment position $\mathbf{r}(n, t)$ of type-A chain under shear and electric fields, eq 6 in the text, can be rewritten as a series of coupled time evolution equations for the amplitudes of the Rouse eigenmodes, $\left\{X_{p}(t), Y_{p}(t), Z_{p}(t)\right\}$ with $p$ $\geq 0$ (defined by eq 8 ). Considering the independence of the internal motion of the chain and the motion of center of mass (described by the Rouse modes with $p \geq 1$ and $p=0$, respectively), we may introduce extended amplitudes $\xi$, with its component being defined by $\xi_{3 p-2}(t)$ $=X_{p}(t), \xi_{3 p-1}(t)=Y_{p}(t)$, and $\xi_{3 p}(t)=Z_{p}(t)$ with $p$ $\geq 1$, and compactly express this series of equations as

$$
\zeta \frac{\mathrm{d}}{\mathrm{d} t} \boldsymbol{\xi}(t)=\mathbf{A} \cdot \boldsymbol{\xi}(t)+\mathbf{B}(t)+\mathbf{D}
$$

Here, $\zeta$ is the segmental friction coefficient and $\mathbf{A}$ is the matrix defined by eqs 11 and 12 in the text. B is a vector with its components defined as the Fourier components of the Brownian force in the $\alpha$ direction, $F_{\mathrm{B}, \alpha}(n, t)(\alpha=x, y, z)$ :

$$
\begin{gathered}
B_{j}(t)=\frac{2}{N} \int_{0}^{N} \mathrm{~d} n F_{B, \alpha}(n, t) \cos \left(\frac{p \pi n}{N}\right) \\
\text { with } j=3 p-2,3 p-1, \text { and } 3 p \text { for } \\
\alpha=x, y, \text { and } z, \text { respectively }
\end{gathered}
$$

D is a vector composed of Fourier components of the electrical force $\left(\mathbf{F}^{\mathrm{E}}\right.$ specified by eq 5$)$,

$$
\begin{aligned}
& D_{j}=0 \quad \text { for } j=3 p-2 \text { and } 3 p, \\
& D_{j}=\frac{2 \tilde{\mu} E}{N}(\cos p \pi-1) \quad \text { for } j=3 p-1
\end{aligned}
$$

We may introduce a matrix $\mathbf{Q}$ that diagonalizes $\mathbf{A}$ to solve eq A7 by a standard method. The solution, obtained for $Y_{p}(t)\left(=\xi_{3 p-1}(t)\right)$ and $X_{p}(t)(=$ $\left.\xi_{3 p-2}(t)\right)$, can be summarized as

$$
\begin{aligned}
& {\left[\begin{array}{c}
X_{p}(t) \\
Y_{p}(t)
\end{array}\right]=\sum_{\beta \geq 1}\left[\begin{array}{l}
Q_{3 p-2, \beta} \\
Q_{3 p-1, \beta}
\end{array}\right]\left\{\eta_{\beta}^{0} \exp \left[-\lambda_{\beta} t\right]\right.} \\
& \left.+\frac{1}{\zeta} \int_{0}^{t} \mathrm{~d} t^{\prime} Q_{\beta, \alpha}^{-1}\left[B_{\alpha}\left(t^{\prime}\right)+D_{\alpha}\left(t^{\prime}\right)\right] \exp \left[-\lambda_{\beta}\left(t-t^{\prime}\right)\right]\right\}
\end{aligned}
$$

Journal of Polymer Science: Part B: Polymer Physics DOI $10.1002 /$ polb 
with

$$
\eta_{\beta}^{0}=\sum_{j \geq 1} Q_{\beta, j}^{-1} \xi_{j}(0)
$$

Here, $Q_{i, j}$ and $Q_{i, j}^{-1}$ are the $i, j$ components of $\mathbf{Q}$ and its inverse matrix $\mathbf{Q}^{-1}$, respectively, and $\Lambda_{\beta}$ is $\beta$-th eigenvalue of $\mathbf{A}$ divided by $\zeta$, that is, $\left\{\mathbf{Q}^{-1} \mathbf{A Q}\right\}_{\alpha \beta}=-\zeta \Lambda_{\beta} \delta_{\alpha \beta}$.

Since the $y$ component of the end-to-end vector is given by $R_{y}(t)=\Sigma_{p \geq 1} Y_{p}(t)\{\cos p \pi-1\}$, the polarization $P_{\mathrm{ss}}(t)$ under steady shear at a time $t$ after imposition of a small constant electric field, $E(>0)$, is straightforwardly obtained from eq $\mathrm{A} 10$ as

$$
\begin{aligned}
P_{\mathrm{ss}}(t ; \dot{\gamma}) & =v \tilde{\mu}\left\langle R_{y}(t+\tau)\right\rangle_{\tau \rightarrow \infty}^{E>0} \\
& =E\left\{\Phi_{\mathrm{ss}}^{\mathrm{un}}(0 ; \dot{\gamma})-\Phi_{\mathrm{ss}}^{\mathrm{un}}(t ; \dot{\gamma})\right\}
\end{aligned}
$$

where $\Phi_{\mathrm{ss}}^{\mathrm{un}}(t ; \dot{\gamma})$ is the unnormalized dielectric relaxation function given by

$$
\Phi_{\mathrm{ss}}^{\mathrm{un}}(t ; \dot{\gamma})=\frac{2 \tilde{\mu}^{2} v}{N \zeta} \sum_{\beta \geq 1} g_{\beta}^{[\Phi]} \exp \left(-\lambda_{\beta} t\right)
$$

with

$$
\begin{aligned}
g_{\beta}^{[\Phi]}=\frac{1}{\lambda_{\beta}} & \left\{\sum_{p \geq 1}(\cos p \pi-1) Q_{3 p-1, \beta}\right\} \\
& \times\left\{\sum_{p^{\prime} \geq 1}\left(\cos p^{\prime} \pi-1\right) Q_{\beta^{\prime}, 3 p^{\prime}-1}^{-1}\right\}
\end{aligned}
$$

In derivation of eq $\mathrm{A} 13$, we have utilized a relationship deduced from the white noise character of the Brownian force, $\left\langle B_{j}(n, t)\right\rangle=0$. (Note that neither the autocorrelation, $\left\langle R_{y}(t+\tau) R_{y}(\tau)\right\rangle_{\tau \rightarrow \infty}$, nor the crosscorrelations, $\left\langle R_{y}(t+\tau) R_{x}(\tau)\right\rangle_{\tau \rightarrow \infty}$ and $\left\langle R_{x}(t+\tau) R_{y}(\tau)\right\rangle_{\tau \rightarrow \infty}$, contribute to $P_{\mathrm{ss}}(t ; \dot{\gamma})$; see eq A12.) Similarly, we can utilize the other relationship deduced from the white noise character, $\left\langle B_{i}(t) B_{j}\left(t^{\prime}\right)\right\rangle=4 \zeta k_{\mathrm{B}} T N^{-1} \delta_{i j} \delta\left(t-t^{\prime}\right)$, to find an expression of the end-to-end vector correlation function from eq A10 with $D_{\alpha}=0$ (in the absence of the electric field),

$$
\begin{aligned}
\Psi_{\mathrm{ss}}(t ; \dot{\gamma}) & \equiv\left\langle R_{y}(t) R_{y}(0)\right\rangle_{\mathrm{ss}}^{E=0} \\
& =\frac{2 k_{\mathrm{B}} T}{N \zeta} \sum_{\beta \geq 1} g_{\beta}^{[\Psi]} \exp \left(-\lambda_{\beta} t\right)
\end{aligned}
$$

with

Journal of Polymer Science: Part B: Polymer Physics DOI $10.1002 /$ polb

$$
\begin{aligned}
g_{\beta}^{[\Psi]} & =\sum_{\beta^{\prime} \geq 1} \frac{2}{\lambda_{\beta}+\lambda_{\beta}^{\prime}}\left\{\sum_{\alpha \geq 1} Q_{\beta, \alpha}^{-1} Q_{\beta^{\prime}, \alpha}^{-1}\right\}\left\{\sum_{p \geq 1}(\cos p \pi-1)\right. \\
& \left.\times Q_{3 p-1, \beta}\right\}\left\{\sum_{p^{\prime} \geq 1}\left(\cos p^{\prime} \pi-1\right) Q_{\beta^{\prime}, 3 p^{\prime}-1}^{-1}\right\} \quad(\mathrm{A} 14 \mathrm{~b})
\end{aligned}
$$

Equations A13 and A14, being identical to eqs 9 and 10 in the text, demonstrate that the dielectric modes and the end-to-end vector fluctuation modes have the same relaxation times $\left(1 / \lambda_{\beta}\right)$ even under steady shear. In contrast, the mode intensities $g_{\beta}^{[\Phi]}$ and $g_{\beta}^{[\Psi]}$ do not exactly coincides with each other (cf. eqs A13b and A14b). However, an argument related to the shear symmetry enables us to deduce the coincidence of $g_{\beta}^{[\Phi]}$ and $g_{\beta}^{[\Psi]}$, at least up to the order of $\dot{\gamma}^{2}$, as explained below.

\section{Perturbation Expansion}

Since the matrix $\mathbf{A}$ (determined by the operator $\Lambda$ in eq 6) depends on the shear rate $\dot{\gamma}$ in general, the diagonalizing matrix $\mathbf{Q}$ and its inverse matrix $\mathbf{Q}^{-1}$ are also dependent on $\dot{\gamma}$. Thus, $\mathbf{Q}$ and $\mathbf{Q}^{-1}$ can be expanded, up to $O\left(\dot{\gamma}^{2}\right)$, as

$$
\begin{aligned}
& \mathbf{Q}=\mathbf{Q}^{(0)}+\dot{\gamma} \mathbf{Q}^{(1)}+\dot{\gamma}^{2} \mathbf{Q}^{(2)}, \\
& \mathbf{Q}^{-1}=\tilde{\mathbf{Q}}^{(0)}+\dot{\gamma} \tilde{\mathbf{Q}}^{(1)}+\dot{\gamma}^{2} \tilde{\mathbf{Q}}^{(2)}
\end{aligned}
$$

Here, the superscripts "(0)", "(1)", and "(2)" stand for the zero-th, first, and second-order expansion coefficients. The zero-th $\mathbf{Q}^{(0)}$ and $\tilde{\mathbf{Q}}^{(0)}$, respectively, are identical to $\mathbf{Q}$ and $\mathbf{Q}^{-1}$ at equilibrium and satisfy an orthogonal relationship (because $\mathbf{A}$ is exactly symmetric at equilibrium),

$$
\tilde{\mathbf{Q}}^{(0)}=\left[\mathbf{Q}^{(0)}\right]^{-1}=\left[\mathbf{Q}^{(0)}\right]^{+}\left(\text {transpose of } \mathbf{Q}^{(0)}\right)
$$

The first and second-order coefficients for $\mathbf{Q}^{-1}$, $\tilde{\mathbf{Q}}^{(1)}$ and $\tilde{\mathbf{Q}}^{(2)}$, are not necessarily identical to the inverse matrices of the coefficients for $\mathbf{Q}, \mathbf{Q}^{(1)}$ and $\mathbf{Q}^{(2)}$. Since $\mathbf{Q} \mathbf{Q}^{-1}=\mathbf{Q}^{-1} \mathbf{Q}=\mathbf{I}=$ order of $\dot{\gamma}^{0}$, these coefficients should satisfy relationships,

$$
\mathbf{Q}^{(0)} \tilde{\mathbf{Q}}^{(1)}+\mathbf{Q}^{(1)} \tilde{\mathbf{Q}}^{(0)}=\tilde{\mathbf{Q}}^{(0)} \mathbf{Q}^{(1)}+\tilde{\mathbf{Q}}^{(1)} \mathbf{Q}^{(0)}=\mathbf{0}
$$

$$
\begin{aligned}
\mathbf{Q}^{(0)} \tilde{\mathbf{Q}}^{(2)}+\mathbf{Q}^{(1)} \tilde{\mathbf{Q}}^{(1)}+\mathbf{Q}^{(2)} \tilde{\mathbf{Q}}^{(0)} & \\
= & \tilde{\mathbf{Q}}^{(0)} \mathbf{Q}^{(2)}+\tilde{\mathbf{Q}}^{(1)} \mathbf{Q}^{(1)}+\tilde{\mathbf{Q}}^{(2)} \mathbf{Q}^{(0)}=\mathbf{0}
\end{aligned}
$$

Considering the above features of $\mathbf{Q}$ and $\mathbf{Q}^{-1}$, we can examine the symmetries of two correlation 
functions, $S_{y x}\left(n, n^{\prime}, t\right) \equiv\left\langle u_{y}(n, t) u_{x}\left(n^{\prime}, 0\right)\right\rangle$ and $S_{y y}\left(n, n^{\prime}, t\right) \equiv\left\langle u_{y}(n, t) u_{y}\left(n^{\prime}, 0\right)\right\rangle$, defined with respect to the $y$ and $x$ components of the bond vector $\mathbf{u}(n, t)(=\partial \mathbf{r}(n, t) / \partial n)$ under steady shear. The symmetries of these functions provide us with useful information for the mode distribution of the unnormalized dielectric relaxation function $\Phi_{\mathrm{ss}}^{\mathrm{un}}(t ; \dot{\gamma})$ and end-to-end vector correlation function $\Psi_{\mathrm{ss}}(t ; \dot{\gamma})$, as explained below.

The steady state is considered to be already achieved at time 0 . Then, from eq A10, $S_{y x}$ and $S_{y y}$ are easily calculated as

$$
\begin{aligned}
& S_{y x}\left(n, n^{\prime}, t\right)=\sum_{p, p^{\prime}} \frac{p p^{\prime} \pi^{2}}{N^{2}} \sin \left(\frac{p \pi n}{N}\right) \\
& \times \sin \left(\frac{p^{\prime} \pi n^{\prime}}{N}\right)\left\langle Y_{p}(t+\tau) X_{p^{\prime}}(\tau)\right\rangle_{\tau \rightarrow \infty}
\end{aligned}
$$

with

$$
\begin{array}{r}
\left\langle Y_{p}(t+\tau) X_{p^{\prime}}(\tau)\right\rangle_{\tau \rightarrow \infty}=\frac{4 k_{\mathrm{B}} T}{N \zeta} \sum_{\beta, \beta^{\prime} \geq 1} Q_{3 p-1, \beta} Q_{3 p^{\prime}-2, \beta^{\prime}} \\
\times\left\{\sum_{\alpha \geq 1} Q_{\beta, \alpha}^{-1} Q_{\beta^{\prime}, \alpha}^{-1}\right\} \frac{\exp \left(-\lambda_{\beta} t\right)}{\lambda_{\beta}+\lambda_{\beta^{\prime}}} \quad(\mathrm{A} 19 \mathrm{~b})
\end{array}
$$

and

$$
\begin{aligned}
& S_{y y}\left(n, n^{\prime}, t\right)=\sum_{p \cdot p^{\prime}} \frac{p p^{\prime} \pi^{2}}{N^{2}} \sin \left(\frac{p \pi n}{N}\right) \\
& \times \sin \left(\frac{p^{\prime} \pi n^{\prime}}{N}\right)\left\langle Y_{p}(t+\tau) Y_{p^{\prime}}(\tau)\right\rangle_{\tau \rightarrow \infty}
\end{aligned}
$$

with

$$
\begin{array}{r}
\left\langle Y_{p}(t+\tau) Y_{p^{\prime}}(\tau)\right\rangle_{\tau \rightarrow \infty}=\frac{4 k_{\mathrm{B}} T}{N \zeta} \sum_{\beta, \beta^{\prime} \geq 1} Q_{3 p-1, \beta} Q_{3 p^{\prime}-1, \beta^{\prime}} \\
\times\left\{\sum_{\alpha \geq 1} Q_{\beta, \alpha}^{-1} Q_{\beta^{\prime}, \alpha}^{-1}\right\} \frac{\exp \left(-\lambda_{\beta} t\right)}{\lambda_{\beta}+\lambda_{\beta^{\prime}}} \quad(\mathrm{A} 20 \mathrm{~b})
\end{array}
$$

A simple consideration of shear symmetry indicates that $S_{y x}$ changes its sign on inversion of the shear direction and is an odd function of $\dot{\gamma}$. In contrast, $S_{y y}$ is invariant to this inversion and is an even function of $\dot{\gamma}$ (which is the case also for $\Phi_{\mathrm{ss}}^{\mathrm{un}}(t ; \dot{\gamma})$ and $\left.\Psi_{\mathrm{ss}}(t ; \dot{\gamma})\right)$. Thus, at any time $t$ and for any indices $p$ and $p^{\prime}$, the terms of the order of $\dot{\gamma}^{2 k}$ and $\dot{\gamma}^{2 k+1}(k=0,1, \ldots)$ obtained after expansion with respect to $\dot{\gamma}$ are required to vanish for $\left\langle Y_{p}(t+\right.$ $\left.\tau) X_{p^{\prime}}(\tau)\right\rangle_{\tau \rightarrow \infty}$ and $\left\langle Y_{p}(t+\tau) Y_{p^{\prime}}(\tau)\right\rangle_{\tau \rightarrow \infty}$, respectively. The relaxation rate is expanded as $\lambda_{\beta}=\lambda_{\beta}^{(0)}+$ $\dot{\gamma}^{2} \lambda_{\beta}^{(2)}+O\left(\dot{\gamma}^{4}\right)$ (even function of $\dot{\gamma}$; cf. eq 13a), and $\mathbf{Q}$ and $\mathbf{Q}^{-1}$ are expanded in the form of eq A15. Considering these expansion forms as well as the orthogonal feature of $\mathbf{Q}^{(0)}$ (eq A16), we can calcu- late the $O\left(\dot{\gamma}^{2 k}\right)$ and $O\left(\dot{\gamma}^{2 k+1}\right)$ terms from eqs A19b and $\mathrm{A} 20 \mathrm{~b}$ to find the conditions satisfying this requirement, as explained below.

The $O\left(\dot{\gamma}^{0}\right)$ term for $\left\langle Y_{p}(t+\tau) X_{p^{\prime}}(\tau)\right\rangle_{\tau \rightarrow \infty}\left(=\left\{2 k_{\mathrm{B}} T /\right.\right.$ $\left.N \zeta\} \Sigma_{\beta \geq 1} Q_{3 p-1, \beta}^{(0)} Q_{3 p^{\prime}-2, \beta}^{(0)}\left\{\lambda_{\beta}^{(0)}\right\}^{-1} \exp \left\{-\lambda_{\beta}^{(0)} t\right\}\right)$ is to vanish for any $p, p^{\prime}$ and at any $t$. Thus, we find

$$
Q_{3 p-1, \beta}^{(0)} Q_{3 p^{\prime}-2, \beta}^{(0)}=0 \quad \text { for any } p, p^{\prime}, \text { and } \beta
$$

Namely, $Q_{3 p-1, \beta}^{(0)}$ and $Q_{3 p^{\prime}-2, \beta}^{(0)}$ at equilibrium cannot simultaneously have nonzero values when their second indices $(\beta)$ are the same. (This feature of $Q_{3 p-1, \beta}^{(0)}$ and $Q_{3 p^{\prime}-2, \beta}^{(0)}$ is necessary to guarantee the Gaussian conformation of the chain at equilibrium.)

The $O\left(\dot{\gamma}^{1}\right)$ term for $\left\langle Y_{p}(t+\tau) Y_{p^{\prime}}(\tau)\right\rangle_{\tau \rightarrow \infty}$, required to vanish at any $t$ and for any $p, p^{\prime}$, is expressed as $\left\{2 k_{\mathrm{B}} T / N \zeta\right\} \Sigma_{\beta \geq 1} h_{p, p^{\prime}, \beta}^{(1)}\left\{\lambda_{\beta}^{(0)}\right\}^{-1} \exp \left(-\lambda_{\beta}^{(0)} t\right)$, with the coefficient $h_{p, p^{\prime}, \beta}^{(1)}$ being given by

$$
\begin{aligned}
& h_{p, p^{\prime}, \beta}^{(1)}=Q_{3 p-1, \beta}^{(0)} Q_{3 p^{\prime}-1, \beta}^{(1)}+Q_{3 p-1, \beta}^{(1)} Q_{3 p^{\prime}-1, \beta}^{(0)} \\
& +\sum_{\beta^{\prime} \geq 1} \frac{2 Q_{3 p-1, \beta}^{(0)} Q_{3 p^{\prime}-1, \beta^{\prime}}^{(0)}}{1+\left\{\lambda_{\beta^{\prime}}^{(0)} / \lambda_{\beta}^{(0)}\right\}}\left\{\sum_{\alpha \geq 1}\left(\tilde{Q}_{\beta, \alpha}^{(0)} \tilde{Q}_{\beta^{\prime}, \alpha}^{(1)}+\tilde{Q}_{\beta, \alpha}^{(1)} \tilde{Q}_{\beta^{\prime}, \alpha}^{(0)}\right)\right\}
\end{aligned}
$$

This $h_{p, p^{\prime}, \beta}^{(1)}$ is required to vanish for any set of the indices, $p, p^{\prime}$, and $\beta$. This is a strong requirement when combined with eq A21, and a "sufficient condition" to meet this requirement is given by

$$
\begin{aligned}
& Q_{3 p-1, \beta}^{(0)} Q_{3 p^{\prime}-1, \beta}^{(1)}+Q_{3 p-1, \beta}^{(1)} Q_{3 p^{\prime}-1, \beta}^{(0)}=0 \\
& \quad \text { for any } p, p^{\prime} \text { and } \beta .
\end{aligned}
$$

and

$$
\sum_{\alpha \geq 1}\left(\tilde{\boldsymbol{Q}}_{\beta, \alpha}^{(0)} \tilde{\boldsymbol{Q}}_{\beta^{\prime}, \alpha}^{(1)}+\tilde{\boldsymbol{Q}}_{\beta, \alpha}^{(1)} \tilde{\boldsymbol{Q}}_{\beta^{\prime}, \alpha}^{(0)}\right)=0
$$

for any $\beta$ and $\beta^{\prime}$

(A24)

Combining eq A24 with eqs A16 and A17, we also find

$$
\tilde{\mathbf{Q}}^{(1)}=\left[\mathbf{Q}^{(1)}\right]^{+} \quad\left(\text { transpose of } \mathbf{Q}^{(1)}\right)
$$

Note that eqs A23 and A24 are not identical to the "sufficient and necessary" condition. For example, eq A24 does not need to hold for particular values of $p$ and $\beta$ giving $Q_{3 p-1, \beta}^{(0)}=0$. However, for general cases, eqs A23 and A24 appear to be close to the "sufficient and necessary" condition for the requirement, $h_{p, p^{\prime}, \beta}^{(1)}=0$ for any $p, p^{\prime}$, and $\beta$.

Considering eqs A16, A21, and A24, we can express the $O\left(\dot{\gamma}^{2}\right)$ term for $\left\langle Y_{p}(t+\tau) X_{p^{\prime}}(\tau)\right\rangle_{\tau \rightarrow \infty}$ as 
$\left\{2 k_{\mathrm{B}} T / N \zeta\right\} \Sigma_{\beta \geq 1} h_{p, p^{\prime}, \beta}^{(2)}\left\{\lambda_{\beta}^{(0)}\right\}^{-1} \exp \left(-\lambda_{\beta}^{(0)} t\right)$, with the coefficient $h_{p, p^{\prime}, \beta}^{(2)}$ being given by

$$
\begin{aligned}
& h_{p, p^{\prime}, \beta}^{(2)}=Q_{3 p-1, \beta}^{(0)} Q_{3 p^{\prime}-2, \beta}^{(2)}+Q_{3 p-1, \beta}^{(1)} Q_{3 p^{\prime}-2, \beta}^{(1)} \\
& +Q_{3 p-1, \beta}^{(2)} Q_{3 p^{\prime}-2, \beta}^{(0)}+\sum_{\beta^{\prime} \geq 1} \frac{2 Q_{3 p-1, \beta}^{(0)} Q_{3 p^{\prime}-2, \beta^{\prime}}^{(0)}}{1+\left\{\lambda_{\beta^{\prime}}^{(0)} / \lambda_{\beta}^{(0)}\right\}} \\
& \times\left\{\sum_{\alpha \geq 1}\left(\tilde{Q}_{\beta, \alpha}^{(0)} \tilde{Q}_{\beta^{\prime}, \alpha}^{(2)}+\tilde{Q}_{\beta, \alpha}^{(1)} \tilde{Q}_{\beta^{\prime}, \alpha}^{(1)}+\tilde{Q}_{\beta, \alpha}^{(2)} \tilde{Q}_{\beta^{\prime}, \alpha}^{(0)}\right)\right\}
\end{aligned}
$$

For the $O\left(\dot{\gamma}^{2}\right)$ term to vanish, this $h_{p, p^{\prime}, \beta}^{(2)}$ is required to vanish for any set of the indices, $p, p^{\prime}$, and $\beta$. This is again a strong requirement when combined with eqs A21 and A23, and the "sufficient" condition for this requirement (close to the "sufficient and necessary" condition giving $h_{p, p^{\prime}, \beta}^{(2)}$ $=0$ for general cases) is given by $Q_{3 p-1, \beta}^{(0)} Q_{3 p^{\prime}-2, \beta}^{(2)}+$ $Q_{3 p-1, \beta}^{(1)} Q_{3 p^{\prime}-2, \beta}^{(1)}+Q_{3 p-1, \beta}^{(2)} Q_{3 p^{\prime}-2, \beta}^{(0)}=0$ (for any $p, p^{\prime}$, and $\beta$ ) and

$$
\sum_{\alpha \geq 1}\left(\tilde{\boldsymbol{Q}}_{\beta, \alpha}^{(0)} \tilde{\boldsymbol{Q}}_{\beta^{\prime}, \alpha}^{(2)}+\tilde{\boldsymbol{Q}}_{\beta, \alpha}^{(1)} \tilde{\boldsymbol{Q}}_{\beta^{\prime}, \alpha}^{(1)}+\tilde{\boldsymbol{Q}}_{\beta, \alpha}^{(2)} \tilde{\boldsymbol{Q}}_{\beta^{\prime}, \alpha}^{(0)}\right)=0
$$

for any $\beta$ and $\beta^{\prime}$

(A27)

Combining eq A27 with eqs A16 and A18, we note

$$
\tilde{\mathbf{Q}}^{(2)}=\left[\mathbf{Q}^{(2)}\right]^{+} \quad\left(\text { transpose of } \mathbf{Q}^{(2)}\right)
$$

We now consider the expansion forms of the unnormalized dielectric relaxation function and the end-to-end vector autocorrelation function up to $O\left(\dot{\gamma}^{2}\right), \quad \Phi_{\mathrm{ss}}^{\mathrm{un}}(t ; \dot{\gamma})=\Phi^{\mathrm{un}(0)}(t)+\dot{\gamma} \Phi_{\mathrm{ss}}^{\mathrm{un}(1)}(t)+$ $\dot{\gamma}^{2} \Phi_{\mathrm{ss}}^{\mathrm{un}(2)}(t)$ and $\Psi_{\mathrm{ss}}(t ; \dot{\gamma})=\Psi^{(0)}(t)+\dot{\gamma} \Psi_{\mathrm{ss}}^{(1)}(t)+$ $\dot{\gamma}^{2} \Psi_{\mathrm{ss}}^{(2)}(t)$. From eqs A13-A16, the zero-th order $\Phi^{\mathrm{un}(0)}(t)$ and $\Psi^{(0)}(t)$ (at equilibrium) are expressed as

$$
\begin{array}{r}
\Phi^{\mathrm{un}(0)}(t)=\frac{2 \tilde{\mu}^{2} v}{N \zeta} \sum_{\beta \geq 1} g_{\beta}^{(0)} \exp \left(-\lambda_{\beta}^{(0)} t\right) \text { and } \\
\Psi^{(0)}(t)=\frac{2 k_{\mathrm{B}} T}{N \zeta} \sum_{\beta \geq 1} g_{\beta}^{(0)} \exp \left(-\lambda_{\beta}^{(0)} t\right)
\end{array}
$$

with

$$
g_{\beta}^{(0)}=\frac{1}{\lambda_{\beta}^{(0)}}\left\{\sum_{p \geq 1}(\cos p \pi-1) Q_{3 p-1, \beta}^{(0)}\right\}^{2}
$$

Namely, $\Phi^{\mathrm{un}(0)}(t)=\left\{\tilde{\mu}^{2} v / k_{\mathrm{B}} T\right\} \Psi^{(0)}(t)$ at any $t$, and these functions are rigorously proportional to each other (which confirms the validity of GreenKubo theorem at equilibrium).

For $\Psi_{\mathrm{ss}}(t ; \dot{\gamma})$, the first order expansion coefficient $\Psi_{\mathrm{ss}}^{(1)}(t)$ vanishes because of eqs A23 and A24. $\left(\Psi_{\mathrm{ss}}(t ; \dot{\gamma})\right.$ is identical to $\int_{0}^{N} \mathrm{~d} n \mathrm{~d} n^{\prime} S_{y y}\left(n, n^{\prime}, t\right)$ and has the same symmetry as $S_{y y}\left(n, n^{\prime}, t\right)$ with respect to $\dot{\gamma}$, so that eqs A23 and A24 deduced from the invariance of $S_{y y}\left(n, n^{\prime}, t\right)$ on inversion of the shear direction automatically give $\Psi_{\mathrm{ss}}^{(1)}(t)$ $=0)$. For $\Phi_{\mathrm{ss}}^{\mathrm{un}}(t ; \dot{\gamma})$, the first-order coefficient is calculated from eqs A14 and A15 as $\Phi_{\mathrm{ss}}^{\mathrm{un}(1)}(t)=$ $\left\{2 \tilde{\mu}^{2} v / N \zeta\right\} \Sigma_{\beta \geq 1} g_{\beta}^{[\Phi](1)} \exp \left(-\lambda_{\beta}^{(0)} t\right)$ with the intensity coefficient $g_{\beta}^{[\Phi](1)}$ being given by

$$
\begin{aligned}
g_{\beta}^{[\Phi](1)}= & \frac{1}{\lambda_{\beta}^{(0)}} \sum_{p, p^{\prime} \geq 1}(\cos p \pi-1)\left(\cos p^{\prime} \pi-1\right) \\
& \times\left(Q_{3 p-1, \beta}^{(0)} Q_{3 p^{\prime}-1, \beta}^{(1)}+Q_{3 p-1, \beta}^{(1)} Q_{3 p^{\prime}-1, \beta}^{(0)}\right)
\end{aligned}
$$

(In derivation of eq A30, we have utilized eq A25.) As seen from eq A23, this $g_{\beta}^{[\Phi](1)}$ vanishes and thus $\Psi_{\mathrm{ss}}^{(1)}(t)=0$ at any $t$. Note also that $g_{\beta}^{[\Phi](1)}=\{2 /$ $\left.\lambda_{\beta}^{(0)}\right\}\left\{\Sigma_{p \geq 1}(\cos p \pi-1) Q_{3 p-1, \beta}^{(0)}\right\} \times\left\{\Sigma_{p^{\prime} \geq 1}\left(\cos p^{\prime} \pi-\right.\right.$ 1) $\left.Q_{3 p^{\prime}-1, \beta}^{(1)}\right\}$ and thus the relationship $g_{\beta}^{[\Phi](1)}=0$ is equivalent to a relationship

$$
\sum_{p^{\prime} \geq 1}\left(\cos p^{\prime} \pi-1\right) Q_{3 p^{\prime}-1, \beta}^{(1)}=0 \quad \text { for any } \beta
$$

Finally, considering eqs A16, A23, A24, A27, A28, and eq A31, we can calculate the second order coefficients for $\Psi_{\mathrm{ss}}(t ; \dot{\gamma})$ and $\Phi_{\mathrm{ss}}^{\mathrm{un}}(t ; \dot{\gamma})$ from eqs A13 and $\mathrm{A} 14$ as

$$
\begin{gathered}
\Psi_{\mathrm{ss}}^{(2)}(t)=\frac{2 k_{\mathrm{B}} T}{N \zeta} \sum_{\beta \geq 1} g_{\beta}^{(2)}(t) \exp \left(-\lambda_{\beta}^{(0)} t\right) \text { and } \\
\Phi_{\mathrm{ss}}^{\mathrm{un}(2)}(t)=\frac{2 \tilde{\mu}^{2} v}{N \zeta} \sum_{\beta \geq 1} g_{\beta}^{(2)}(t) \exp \left(-\lambda_{\beta}^{(0)} t\right)
\end{gathered}
$$

with

$$
\begin{aligned}
& g_{\beta}^{(2)}=\frac{2}{\lambda_{\beta}^{(0)}}\left\{\sum_{p \geq 1}(\cos p \pi-1) Q_{3 p-1, \beta}^{(0)}\right\} \\
& \times\left\{\sum_{p^{\prime} \geq 1}\left(\cos p^{\prime} \pi-1\right) Q_{3 p^{\prime}-1, \beta}^{(2)}\right\}-\frac{1}{\lambda_{\beta}^{(0)}}\left\{\frac{\lambda_{\beta}^{(2)}}{\lambda_{\beta}^{(0)}}+\lambda_{\beta}^{(2)} t\right\} \\
& \times\left\{\sum_{p \geq 1}(\cos p \pi-1) Q_{3 p-1, \beta}^{(0)}\right\}^{2}(\mathrm{~A} 32 \mathrm{~b})
\end{aligned}
$$

Thus, $\Phi_{\mathrm{ss}}^{\mathrm{un}(2)}(t ; \dot{\gamma})=\left\{\tilde{\mu}^{2} v / k_{\mathrm{B}} T\right\} \Psi_{\mathrm{ss}}^{(2)}(t ; \dot{\gamma})$ at any $t$. 
From the above results, we find the validity of the Green-Kubo theorem, $\Phi_{\mathrm{ss}}^{\mathrm{un}}(t ; \dot{\gamma})=\left\{\tilde{\mu}^{2} v /\right.$ $\left.k_{\mathrm{B}} T\right\} \Psi_{\mathrm{ss}}(t ; \dot{\gamma})$, up to the order of $\dot{\gamma}^{2}$. This conclusion is based on the "sufficient" condition deduced from the symmetries of $S_{y x}\left(n, n^{\prime}, t\right)$ and $S_{y y}\left(n, n^{\prime}, t\right)$ with respect to $\dot{\gamma}$ under steady shear, and we cannot rigorously rule out an anomalous type of dynamics that allows the entangled polymer chains to violate the Green-Kubo theorem up to $O\left(\dot{\gamma}^{2}\right)$. However, we expect that the entanglement dynamics is equivalent to the Rouse dynamics being modified due to the topological constraint and this constraint does not severely change the Rouse Hamiltonian. The Rouse Hamiltonian ensures the validity of the GreenKubo theorem. Thus, the Green-Kubo theorem quite possibly holds for actual entangled chains under steady shear at least up to $O\left(\dot{\gamma}^{2}\right)$.

\section{APPENDIX C: FORMAL THEORY OF LINEAR RESPONSE IN NONEQUILIBRIUM STEADY STATE}

Recently, formal theories of linear response under specific nonequilibrium steady state conditions have been developed on the basis of Langevin equation..$^{20,21}$ These theories are mathematically exact, but the current formulation is applicable only to idealized, simple systems. For realistic (and complicated) systems such as the system of type-A polymer chains, it is difficult to analyze quantitatively the linear response under steady shear within the context of those formal theories. Nevertheless, it is informative to utilize the theories to compare qualitatively the linear responses of idealized and realistic materials, colloidal particles driven by a constant force along a circular orbit ${ }^{20,21}$ and type-A chains under steady shear, thereby examining the characteristic feature of the type-A chains. This comparison is made below.

We first consider the colloidal particle on a circular orbit driven by a constant force along this orbit ${ }^{20,21}$ : the particle position is measured with a curvilinear coordinate $x$ that has multiple values according to the number of round travels along the orbit. ( $x$ increases by the orbit length $L_{\mathrm{o}}$ on each round trip of the particle in a positive direction.) Utilizing this coordinate, we can model the particle motion as one-dimensional motion along an infinite axis $(-\infty<x<\infty)$. The particle is subjected to the constant driving force $F_{0}(>0)$ as well as the Brownian force $F^{\mathrm{B}}(t)$ hav- ing the white noise character $\left(\left\langle F^{\mathrm{B}}(t) F^{\mathrm{B}}\left(t^{\prime}\right)\right\rangle=\right.$ $2 \zeta k_{\mathrm{B}} T \delta\left(t-t^{\prime}\right)$ with $\zeta=$ friction coefficient of the particle) and a force due to a periodic potential $U(x)$ having the periodicity $L\left(=L_{0} / K\right.$ with $K=$ a positive integer). The Langevin equation for the particle position $x(t)$ is given $b^{20}$

$$
\zeta \dot{x}(t)=-\frac{\mathrm{d} U(x)}{\mathrm{d} x}+F_{0}+F^{\mathrm{B}}(t)
$$

It can be straightforwardly shown that a constant probability current $J_{0}$ emerges in the steady state and thus the thermal equilibrium is not achieved in this state. The velocity autocorrelation function of the particle (related to the particle diffusion) is calculated from eq A33, as explained below.

In general, a linear response function $\Theta$ of a physical quantity $A$ against a field conjugate to a physical quantity $B$ can be rigorously calculated with a standard method ${ }^{27}$ as

$$
\Theta(t-s)=\beta\langle A(t) \dot{B}(s)\rangle_{\mathrm{ss}}-\beta\left\langle A(t) \frac{\partial B(s)}{\partial x(s)} v\right\rangle_{\mathrm{ss}}
$$

where $\beta=1 / k_{\mathrm{B}} T, v \equiv J_{0} / f_{\mathrm{ss}}$ with $f_{\mathrm{ss}}$ being the steady state probability distribution function, and the subscript "ss" stands for the quantity in the steady state in the absence of the field. The first term in the right hand side of eq A34 is of the usual Green-Kubo form and the second term gives a nonequilibrium correction in the steady state. The velocity autocorrelation function of the driven particle is straightforwardly obtained by substituting $A=\dot{x}-\langle\dot{x}\rangle_{\mathrm{ss}}$ and $B=x$ in eq A34 as

$$
\begin{aligned}
\Theta(t-s)= & \beta\left\langle\left(\dot{x}(t)-\langle\dot{x}\rangle_{\mathrm{ss}}\right)\left(\dot{x}(s)-\langle\dot{x}\rangle_{\mathrm{ss}}\right)\right\rangle_{\mathrm{ss}} \\
& -\beta\left\langle\left(\dot{x}(t)-\langle\dot{x}\rangle_{\mathrm{ss}}\right)\left(v-\langle\dot{x}\rangle_{\mathrm{ss}}\right)\right\rangle_{\mathrm{ss}}
\end{aligned}
$$

It has been shown that the second term (correction term) is not negligible compared to the first term $^{20,21}$ partly because the average $\langle\dot{x}\rangle_{\mathrm{ss}}$ has a nonzero value. In fact, the asymptotic form of the second term at short times $(t-s \rightarrow 0)$, obtained after integration by parts, is expressed in terms of the constant driving force $F_{0}$, constant probability current $J_{0}$, and the potential periodicity $L \mathrm{as}^{20}$

$$
\begin{gathered}
-\beta\left\langle\left(\dot{x}(t)-\langle\dot{x}\rangle_{\mathrm{ss}}\right)\left(v-\langle\dot{x}\rangle_{\mathrm{ss}}\right)\right\rangle_{\mathrm{ss}} \rightarrow-\beta F_{0} J_{0} L+\langle\dot{x}\rangle_{\mathrm{ss}}^{2} \\
+O(t-s) \cong-\beta F_{0} J_{0} L \quad(\text { for } t-s \rightarrow 0) \quad(\mathrm{A} 36)
\end{gathered}
$$

As can be noted from eqs A35 and A36, the second term has a large absolute value comparable to the first term at short times, ${ }^{20}$ and both terms decay 
to zero at long times $(t-s \rightarrow \infty)$ to result in the relaxation of the response function $\Theta$. In other words, the second term has a large contribution to $\Theta$ at short times and thus this contribution survives in the whole process of the relaxation of $\Theta$. For this reason, the Green-Kubo theorem does not hold for the particle driven by the constant force. (It should be also noted that the Green-Kubo theorem recovers its validity if the local velocity is appropriately redefined. ${ }^{20}$ This validity is consistent with the linear response theory of Evans et al. ${ }^{17,19}$ for a thermostatted particle system under steady shear.)

Now, we turn our attention to the system of type-A chains under steady shear. The Langevin equation for the position of $n$-th segment $\mathbf{r}(n, t)$ (in the absence of the electric field) can be written as

$$
\zeta \dot{\mathbf{r}}(n, t)=-\frac{\partial U(\{\mathbf{r}(n, t)\})}{\partial \mathbf{r}(n, t)}+\zeta \dot{\boldsymbol{\Gamma}} \cdot \mathbf{r}(n, t)+\mathbf{F}^{\mathrm{B}}(n, t)
$$

Here, $\zeta$ is the segmental friction coefficient, $U$ is the interaction potential determined by the positions of all segments $\{\mathbf{r}(n, t)\}, \dot{\Gamma}$ is the shear rate tensor (given by eq 7 ), and $\mathbf{F}^{\mathrm{B}}(n, t)$ is the Brownian force acting on the $n$th segment at time $t$. Note that the Langevin equation utilized in the text (eq 6) is obtained by linearizing the first term in the right hand side of eq A37 (and adding the force due to the electric field).

The linear response function $\Theta$ of a physical quantity $A$ of the type-A chain system against a field conjugate to a physical quantity $B$ can be formulated similarly to the one-dimensional case (eq A34) as

$$
\begin{aligned}
\Theta(t-s)= & \beta\langle A(t) \dot{B}(s)\rangle_{\mathrm{ss}} \\
& -\beta\left\langle A(t) \sum_{n} \frac{\partial B(s)}{\partial \mathbf{r}(n, s)} \cdot \mathbf{v}(n, s)\right\rangle_{\mathrm{ss}}
\end{aligned}
$$

In eq $\mathrm{A} 38$, we have introduced, in analogy to the one dimensional case, the local velocity $\mathbf{v}(n) \equiv$ $\mathbf{J}(n) / f_{\mathrm{ss}}$, where $\mathbf{J}(n)$ denotes the steady state probability flux at the position of the $n$-th segment and $f_{\mathrm{ss}}$ is the steady state probability distribution function. The rheodielectric response function of the type-A chains defined in the shear gradient direction is obtained by replacing both of $A$ and $B$ in eq A38 by the end-to-end dipole in this direction, $\tilde{\mu} R_{y}$ :

Journal of Polymer Science: Part B: Polymer Physics DOI 10.1002/polb

$$
\begin{aligned}
\Theta(t-s) & =\beta \tilde{\mu}^{2}\left\langle R_{\mathrm{y}}(t) \dot{R}_{\mathrm{y}}(s)\right\rangle_{\mathrm{ss}} \\
& -\beta \tilde{\mu}^{2}\left\langle R_{\mathrm{y}}(t) \sum_{n} \frac{\partial R_{\mathrm{y}}(s)}{\partial \mathbf{r}(n, s)} \cdot \mathbf{v}(n, s)\right\rangle_{\mathrm{ss}}
\end{aligned}
$$

This $\Theta(t)$ is contributed from not only the GreenKubo term (first term in the right hand side) but also the nonequilibrium correction term (second term), which is formally similar to the case of driven particle (eq A35). However, we also note a quantitative difference between eqs. A39 and A35. The asymptotic form of the correction term in eq A39 at short times $(t-s \rightarrow 0)$ is found to be

$$
\begin{aligned}
-\beta \tilde{\mu}^{2}\left\langle R_{\mathrm{y}}(t) \sum_{n} \frac{\partial R_{\mathrm{y}}(s)}{\partial \mathbf{r}(n, s)} \cdot \mathbf{v}(n, s)\right\rangle_{\mathrm{ss}} \rightarrow \\
-\frac{\beta \tilde{\mu}^{2}}{2}\left\langle\sum_{n} \frac{\partial\left\{R_{\mathrm{y}}(s)\right\}^{2}}{\partial \mathbf{r}(n, s)} \cdot \mathbf{v}(n, s)\right\rangle_{\mathrm{ss}}+O(t-s) \\
=O(t-s) \rightarrow 0 \quad(\text { for } t-s \rightarrow 0) \quad(\mathrm{A}
\end{aligned}
$$

In derivation of eq $\mathrm{A} 40$, we have made integration by parts and utilized a relationship $\sum_{n} \frac{\partial \mathbf{J}(n, s)}{\partial \mathbf{r}(n, s)}=0$ (indicating the lack of divergence of the steady state probability flux). As can be noted from eqs. A39 and A40, at short times the Green-Kubo term is much larger, in magnitude, compared with the correction term $(\sim 0)$, and both term decays at long times to result in the relaxation of the response function $\Theta$. Thus, the nonequilibrium correction seems to have only minor contribution to $\Theta$ of the type-A chains in the entire range of time, although this correction may increase its magnitude to have a somewhat larger contribution at intermediate times.

The above discussion suggests that the nonequilibrium correction is minor for the rheodielectric relaxation function of the type-A chains under steady shear. This result lends support to the (approximate) validity of the Green-Kubo theorem for the rheodielectric response of typeA chains discussed in the text on the basis of the linearized Langevin equation (eq 6). Of course, the above analysis is approximate, and it is strongly desired to develop a linear response theory that enables a quantitative analysis of the actual rheodielectric behavior of type-A chains. At the same time, we believe that the analysis presented in this paper has an importance as the first attempt in the absence of such a theory. 
Finally, it is informative to consider the ori$\operatorname{gin}(\mathrm{s})$ of the difference between the type-A chains and the driven particle, the former satisfactorily obeying while the latter violating the Green-Kubo theorem. This difference may be partly related a coupling incorporated in the Langevin equation. Namely, the particle position $x$, the quantity averaged in the response function $\Theta$, is directly coupled with the driving force $F_{0}$ and frictional force $\zeta \dot{x}$ in eq A33, while in eq A37 the $y$ component of the end-to-end vector $R_{y}$ of the type-A chain (averaged in $\Theta$ ) is not directly coupled with the frictional force due to steady shear acting in the $x$ direction. This difference could lead to the difference of the validity of the Green-Kubo theorem. (In fact, the analysis based on eq A37 as well as eq 6 shows that the theorem severely fails for the crosscorrelation between $R_{y}$ and $R_{x}$, the latter being coupled directly with the frictional force due to steady shear.) In addition, the average $\langle\dot{x}\rangle_{\mathrm{ss}}$ for the particle has a nonzero value while the average $\left\langle\dot{R}_{y}\right\rangle_{\text {ss }}$ for the type-A chains vanishes, which may also contribute to the difference between the particle and type-A chains.

\section{APPENDIX D: NAPLES SIMULATION}

Masubuchi et al. ${ }^{25}$ developed a method of coarse-grained molecular simulation (NAPLES) for entangled chains based on the primitive chain network model. In this model, the network is composed of Gaussian chains that are pair-wise connected at temporary slip links (that represent entanglements), and the chains are allowed to slide along the array of slip links according to a balance of forces acting on each portion of the chains between slip links (this portion is referred as a strand); the forces considered are the frictional force from a medium, the elastic force due to the conformational entropy of a strand, the Brownian force activating diffusion, and the thermodynamic force reducing any spatial gradient of segment density (a force due to chemical potential). The segment number in each strand is also allowed to fluctuate according to a similar force balance. The slip link fluctuates/moves in space following the force balance for the strands. In addition, the slip link penetrated by a strand at the chain end is occasionally removed/created on decrease/ increase of the segment number in this strand below/above critical values. Mathematical for- mulation of these kinetic changes has been explained elsewhere. ${ }^{25,28-30}$

Masubuchi et al. ${ }^{25,28-30}$ showed that the NAPLES simulation describes considerably well the linear and nonlinear viscoelastic behavior of entangled homopolymers including PI (and the dielectric behavior of linear PI at equilibrium). For PI under steady shear, they also conducted a preliminary NAPLES simulation ${ }^{26}$ incorporating the so-called convective constraint release $(\mathrm{CCR})^{31}$ and hidden entanglement appearance $(\mathrm{HEA})^{32}$ mechanisms to calculate the rheodielectric response. The CCR mechanism represents the release of the entanglement for a given chain due to retraction of surrounding chains under fast shear (that is still slower than their Rouse motion), and the HEA mechanism considers that a chain that was neighboring to but not entangled with the given chain before occurrence of CCR is hooked by that chain after CCR. In the rheodielectric simulation, Masubuchi et al. $^{26}$ put positive and negative charges at respective ends of the entangled linear chains and directly evaluated, by monitoring the spatial distribution of these charges, the macroscopic polarization $P_{\mathrm{ss}}(t)$ induced by a weak electric field $E$. These charges are equivalent to a sum of type-A dipoles of the chain. Thus, the simulation well mimicked the actual rheodielectric experiments for PI under steady shear. In the simulation, the macroscopic dielectric relaxation function directly obtained from this $P_{\mathrm{ss}}(t)$ well agreed with the correlation function of the endto-end vector $R_{y}$ in the shear gradient direction, ${ }^{26}$ lending support to eqs $14 \mathrm{a}$ and $14 \mathrm{~b}$ in the text.

In this study, we have again conducted the NAPLES simulation (with the CCR and HEA mechanisms) for the rheodielectric behavior of entangled PI under steady shear as well as LAOS. Among several versions of NAPLES with minor differences, the simulation was performed with the code used in recent studies showing quantitative prediction of linear ${ }^{28-30}$ and nonlinear $^{30}$ viscoelastic behavior. The CCR and HEA mechanisms were incorporated in the simulation, and the HEA parameter $l_{\mathrm{c}}^{2}$, specifying a spatial range where the entanglement can be created, was set at 2.0 , as done in the previous study. ${ }^{26}$

The simulation was made for monodisperse linear chains each having 9.6 entanglement segments on average at equilibrium. The simulation box size and segment density were $(16 a)^{3}$ 
and $10 a^{-3}$, respectively, with $a$ being an average length of entanglement segment. This box was subjected to steady shear and/or LAOS, both being generated with SLLOD and Lees-Edwards boundary condition. The steady shear rate as well as the LAOS frequency and amplitude in the simulation mimicked the actual experimental conditions. ${ }^{7,11}$ For each chain in the box, a positive charge $\tilde{\mu}$ was attached to one end, and a negative charge $-\tilde{\mu}$ to the other end. (These charges are equivalent to the type-A dipoles summed along the chain backbone.) The chains under steady shear or LAOS were also subjected to a small oscillatory electric field $\left(E(t)=E_{0} \sin \right.$ $\omega t$ ) in the shear gradient direction. The resulting, macroscopic polarization $P(t)$ was directly evaluated from the spatial distribution of the chain ends, and the dielectric loss was calculated from this $P(t)$. The end-to-end vector correlation function and the mean-square end-to-end distance $\left\langle R_{y}^{2}\right\rangle_{\text {LAOS }}$, respectively, were also evaluated under steady shear and LAOS. The simulated results are shown in Figures 1-3 in this paper. Although the simulated dependence of the terminal dielectric relaxation time $\tau_{\varepsilon}$ on the steady shear rate/LAOS amplitude is stronger than the experimental observation, the general trends of the rheodielectric behavior are well captured by the simulation; see Figures 1-3.

\section{REFERENCES AND NOTES}

1. Watanabe, H. Prog Polym Sci 1999, 24, 12531403.

2. McLeish, T. C. B. Adv Phys 2002, 51, 1379-1527.

3. Matsumiya, Y.; Watanabe, H.; Inoue, T.; Osaki, K.; Yao, M. L. Macromolecules 1998, 31, 79737975 .

4. (a) Watanabe, H.; Sato, T.; Hirose, M.; Osaki, K.; Yao, M. L. Rheol Acta 1998, 37, 519-527; (b) Watanabe, H.; Sato, T.; Hirose, M.; Osaki, K.; Yao, M. L. Rheol Acta 1999, 38, 100-107.

5. Watanabe, H.; Sato, T.; Matsumiya, Y.; Inoue, T.; Osaki, K. Nihon Reoroji Gakkaishi (J Soc Rheol Japan) 1999, 27, 121-125.

6. Watanabe, H.; Matsumiya, Y.; Kakiuchi, M.; Aoki, Y. Nihon Reoroji Gakkaishi (J Soc Rheol Japan) 2001, 29, 77-80.

7. Watanabe, H.; Ishida, S.; Matsumiya, Y. Macromolecules 2002, 35, 8802-8818.
8. Watanabe, H.; Matsumiya, Y.; Inoue, T. J Phys Condensed Matter 2003, 15, S909-S921.

9. Matsumiya, Y.; Balsara, N. P.; Kerr, J. B.; Inoue, T.; Watanabe, H. Macromolecules 2004, 37, 544553.

10. Watanabe, H.; Matsumiya, Y.; Inoue, T. Macromol Symp 2005, 228, 51-70.

11. Höfl, S.; Kremer, F.; Spiess, H. W.; Wilhelm, M.; Kahle, S. Polymer 2006, 47, 7282-7288.

12. Capaccioli, S.; Prevosto, D.; Best, A.; Hanewald, A. Pakula, T. J Non-Crystal Solids 2007, 353, 4267-4272.

13. Cole, R. J Chem Phys 1965, 42, 637-643.

14. Kubo, R. J Phys Soc Japan 1957, 12, 570-586.

15. Evans, D.; Morris, P. Statistical Mechanics of Nonequilibrium Liquids; Academic Press: London, 1990; Chapter 4, p 94.

16. Adachi, K.; Kotaka, T. Prog Polym Sci 1993, 18, 582-622.

17. Evans, D. J Phys Rev A 1991, 44, 3630-3632.

18. Evans, D.; Morris, P. Statistical Mechanics of Nonequilibrium Liquids; Academic Press: London, 1990; Chapter 5, p 114.

19. Evans, D. J.; Baranyai, A.; Sarman, S. Mol Phys 1992, 76, 661-667.

20. Speck, T.; Seifert, U. Europhys Lett 2006, 74, 391-396.

21. Harada, T.; Sasa, S. Phys Rev Lett 2005, 95, 130602

22. Watanabe, H.; Urakawa, O.; Kotaka, T. Macromolecules 1993, 26, 5073-5083.

23. Watanabe, H.; Urakawa, O.; Kotaka, T. Macromolecules 1994, 27, 3525-3536.

24. Watanabe, H.; Matsumiya, Y.; Osaki, K.; Yao, M.-L. Macromolecules 1998, 31, 7538-7545.

25. Masubuchi, Y.; Takimoto, J.; Koyama, K.; Ianniruberto, G.; Greco, F.; Marrucci, G. J Chem Phys 2001, 115, 4387-4394.

26. Masubuchi, Y.; Watanabe, H.; Ianniruberto, G.; Greco, F.; Marrucci, G. Nihon Reoroji Gakkaishi (J Soc Rheol Japan) 2004, 32, 197-202.

27. Risken, H. The Fokker-Planck Equation, 2nd ed.; Springer-Verlag: Berlin, 1989; Chapter 7, p 163.

28. Masubuchi, Y.; Ianniruberto, G.; Marrucci, G.; Greco, F. J Chem Phys 2003, 119, 6925-6930.

29. Masubuchi, Y.; Ianniruberto, G.; Greco, F.; Marrucci, G. J Non-Newtonian Fluid Mech 2008, 149, 87-92.

30. Masubuchi, Y. Nihon Reoroji Gakkaishi (J Soc Rheol Japan) 2006, 34, 275-282.

31. Ianniruberto, G.: Marrucci, G. J Non-Newtonian Fluid Mech 1996, 65, 241-246.

32. Ianniruberto, G.: Marrucci, G. J Non-Newtonian Fluid Mech 2000, 95, 363-374. 